$\mathrm{PM} / 96-35$

KA-TP-28-1996

December 1996

\title{
The coupling of the lightest SUSY Higgs boson to two photons in the decoupling regime
}

\author{
A. DJouadi ${ }^{1,2}$, V. Driesen ${ }^{2}$, W. Hollik ${ }^{2}$ And J.I. Illana ${ }^{2}$ \\ ${ }^{1}$ Laboratoire de Physique Mathématique et Théorique, UPRES-A 5032, \\ Université de Montpellier II, F-34095 Montpellier Cedex 5, France. \\ ${ }^{2}$ Institut für Theoretische Physik, Universität Karlsruhe, \\ D-76128 Karlsruhe, FR Germany.
}

\begin{abstract}
We analyze the contribution of the SUSY particles to the coupling of the lightest Higgs boson to two photons in supersymmetric theories. We discuss to what extent these contributions can be large enough to allow for a discrimination between the lightest SUSY and the standard Higgs particles in the decoupling limit where all other Higgs bosons are very heavy and no supersymmetric particle has been discovered at future colliders. We find that only chargino and top squark loops can generate a sizeable difference between the standard and the SUSY Higgs-photon couplings. For masses above $250 \mathrm{GeV}$, the effect of chargino loops on the twophoton width is however smaller than $\sim 10 \%$ in the entire SUSY parameter space. Top squarks heavier than $250 \mathrm{GeV}$ can induce deviations larger than $10 \%$ only if their couplings to the Higgs boson are large. Since top squark contributions can be sizeable, we derive the two-loop QCD correction to squark loops and show that they are well under control.
\end{abstract}




\section{Introduction}

In supersymmetric (SUSY) theories, the Higgs sector is extended to contain at least two isodoublets of scalar fields. In the minimal version, the Minimal Supersymmetric Standard Model (MSSM), this leads to the existence of five Higgs particles: two CP-even Higgs bosons $h$ and $H$, a $\mathrm{CP}-$ odd or pseudoscalar Higgs boson $A$, and two charged Higgs particles $H^{ \pm}$[1]. Besides the four masses, two additional parameters are needed to describe the Higgs sector at tree-level: $\tan \beta$ the ratio of the two vacuum expectation values and a mixing angle $\alpha$ in the $\mathrm{CP}$-even sector. However, only two of these parameters are independent, and choosing the pseudoscalar mass $M_{A}$ and $\tan \beta$ as inputs, the structure of the MSSM Higgs sector is entirely determined.

If the pseudoscalar mass $M_{A}$ is very large, $M_{A}^{2} \gg M_{Z}^{2}$, the pattern of Higgs masses is quite regular. The heavy $\mathrm{CP}$-even, $\mathrm{CP}$-odd and charged Higgs bosons are nearly mass degenerate, $M_{H} \simeq M_{H^{ \pm}} \simeq M_{A}$, while the lightest $\mathrm{CP}$-even $h$ particle reaches its maximal mass value. At tree level, this value is simply a function of $\tan \beta, M_{h}^{\max }=M_{Z}|\cos 2 \beta| \leq$ $M_{Z}$. However when including the radiative corrections [2, 3] which grow as the fourth power of the top mass and logarithmically with the common squark mass, the upper bound is shifted upwards, $M_{h}^{\max } \simeq 130 \mathrm{GeV}$. In this so called decoupling limit $\llbracket$, which in practice is reached for $M_{A} \sim 300 \mathrm{GeV}$, the lightest SUSY Higgs boson $h$ has almost the same properties as the SM Higgs particle $H^{0}$ and the MSSM and SM Higgs sectors look practically the same, with one light Higgs boson with a mass below $\sim 130 \mathrm{GeV}$.

In the case where no genuine SUSY particle and no additional Higgs boson has been discovered at future high-energy colliders, the task of discriminating between the lightest SUSY and the standard Higgs bosons, and therefore between the MSSM and the SM, in the decoupling limit is challenging. Indeed, since both have almost the same couplings to fermions and vector bosons, the production rates and the decay branching ratios [when SUSY Higgs decays are kinematically not allowed] are practically identical.

Only indirectly that one can distinguish between the two models: if the SM is extended to the GUT scale, the value $m_{t} \simeq 175 \mathrm{GeV}$ requires a Higgs boson heavier than $M_{H^{0}} \gtrsim 130$ $\mathrm{GeV}$ [5] in order that the vacuum remains stable; since in the MSSM, $M_{h}$ is constrained to be lighter than $\sim 130 \mathrm{GeV}$, the measured Higgs mass will allow to discriminate between the SM and MSSM scenarios [6]. However, one can assume that new physics beyond the $\mathrm{SM}$ exists at a scale $\Lambda \lesssim 10 \mathrm{TeV}$ and in this case, Higgs masses in the range $M_{H^{0}} \sim M_{Z}$ will be still allowed. Furthermore, the SUSY Higgs mass bound $M_{h} \lesssim 130 \mathrm{GeV}$ is valid only in the MSSM: for more general SUSY scenarios where the Higgs sector is even more

complicated [for instance in the NMSSM where an additional Higgs singlet is added], the upper bound on the lightest Higgs mass from triviality can be extended to $M_{h}^{\max } \sim 150$ $\mathrm{GeV}$ [7], leaving a room for an overlap between the allowed $h$ and $H^{0}$ masses. 
A more "direct" way to discriminate between the standard and the lightest SUSY Higgs particles is to look at loop induced Higgs boson couplings such as the $\Phi g g$ [8], $\Phi Z_{\gamma}$ [9] and $\Phi \gamma \gamma$ [10] couplings, $\Phi \equiv h$ or $H^{0}$. In the SM, these couplings are mediated by heavy quark and $W$ boson loops [only quark loops for the $H^{0} g g$ coupling]: since their couplings to the Higgs boson grow with the mass, they balance the decrease of the triangle amplitude with increasing loop mass, and these particles do not decouple even for masses much larger than $M_{H^{0}}$. In supersymmetric theories, additional contributions will be induced by loops with charged Higgs bosons, charginos and sfermions; Fig. 1. However, since the SUSY particles do not couple to the Higgs boson proportionally to their masses, their contributions are expected to be rather small for large masses. For very heavy SUSY particles, the loop induced vertices reduce to their SM values and again, no distinction between the SM and the MSSM can be made.

The $\Phi g g$ vertex can be measured in the main Higgs production process $g g \rightarrow \Phi$ at hadron colliders, or via the branching ratio $\mathrm{BR}(\Phi \rightarrow g g)$ with the Higgs boson produced at $e^{+} e^{-}$colliders. At the LHC, the determination of the cross section $\sigma(g g \rightarrow \Phi)$ to the level of ten percent is rather difficult, due to uncertainties from the QCD corrections [which at next-to-leading order are very large [11, 12], increasing the cross section by almost a factor of two] and to a lesser extent from the parton densities. The branching ratio $\operatorname{BR}(\Phi \rightarrow g g)$ is of the order of a few percent for $M_{\Phi} \sim 100 \mathrm{GeV}$, and its measurement at $e^{+} e^{-}$colliders with an accuracy of more than a few ten percent is also very difficult due to the contamination from charm and bottom quarks [13.

The $\Phi Z \gamma$ vertex can be measured in the decay $Z \rightarrow \Phi \gamma$ at LEP and SLC if $M_{\Phi}<M_{Z}$ or in the reverse decay $\Phi \rightarrow Z \gamma$ if $M_{\Phi}>M_{Z}$ with the Higgs boson produced in the $g g \rightarrow \Phi$ fusion mechanism at the LHC. However the rates are very small, $\operatorname{BR}(Z \rightarrow \Phi \gamma) \lesssim 10^{-6}$ and $\operatorname{BR}\left(\Phi \rightarrow Z \gamma \rightarrow l^{+} l^{-} \gamma\right) \lesssim 10^{-4}$, leading to only a few events at LEP or the LHC and making the determination of the $\Phi Z \gamma$ vertex with a reasonable accuracy very difficult. At future $e^{+} e^{-}$colliders with the expected integrated luminosities $\int \mathcal{L} \sim 50 \mathrm{fb}^{-1}$, running a few months on the $Z$ resonance would allow to obtain a large sample of $Z \rightarrow \Phi \gamma$ events if $M_{\Phi}<M_{Z}$; a precise measurement of the $\Phi Z \gamma$ coupling would be possible in this case [14].

The prospects for measuring the loop induced $\Phi \gamma \gamma$ vertex are as follows:

(i) At the LHC the production rate for light Higgs bosons is very large, $\sigma(g g \rightarrow \Phi) \sim$ $100 \mathrm{pb}$ [15], and despite of the small branching ratio $\operatorname{BR}(\Phi \rightarrow \gamma \gamma) \sim 10^{-3}$, one would still have $\mathcal{O}\left(10^{3}\right) \gamma \gamma$ events after filtering out most of the background events, if the luminosity is high enough, $\mathcal{L} \sim 10^{34} \mathrm{~cm}^{-2} \mathrm{~s}^{-1}$. However, as discussed earlier, besides the uncertainties from the parton densities, the theoretical prediction of the production cross sections is affected by large uncertainties from higher QCD corrections. Since one measures only $\sigma \times \mathrm{BR}$, a clean extraction of the $\Phi \rightarrow \gamma \gamma$ width will be rather difficult. 
(ii) At $e^{+} e^{-}$colliders $\rrbracket$, the main production mechanisms for Higgs particles are the bremsstrahlung process $e^{+} e^{-} \rightarrow \Phi Z$ and the $W W$ fusion process $e^{+} e^{-} \rightarrow W^{*} W^{*} \rightarrow \Phi \bar{\nu}_{e} \nu_{e}$ [15]. At energies in the range of $\sqrt{s} \sim 500 \mathrm{GeV}$, the cross sections are around $100 \mathrm{fb}$ for each process; even for integrated luminosities of $\sim 50 \mathrm{fb}^{-1}$ one would have only a few $\Phi \rightarrow \gamma \gamma$ events, a sample which does not allow a precise measurement. At higher energies the cross section for the $W W$ fusion mechanism increases logarithmically: at $\sqrt{s} \sim 1.5$ $\mathrm{TeV}$ and with $\int \mathcal{L} \sim 200 \mathrm{fb}^{-1}$, one would have $\mathcal{O}(100)$ events allowing for a decent measurement. However, if no SUSY particles have been found at this energy, their effect on the $\Phi \rightarrow \gamma \gamma$ width will probably be too small to be visible.

(iii) The most promising way to have access to the $\Phi \gamma \gamma$ coupling is via the single Higgs production in the fusion process $\gamma \gamma \rightarrow \Phi[18-22]$, with the photons generated by Compton-back scattering of laser light [23]. One can tune the energy of the $\gamma \gamma$ collider such as to produce the Higgs boson as a resonance in the $s$-channel. If the luminosity of the $\gamma \gamma$ collider is of the same order as the luminosity of the original $e^{+} e^{-}$collider, large production rates can be obtained. A measurement of the $\Phi \rightarrow \gamma \gamma$ partial decay width with a precision of the order of $10 \%$ could be feasible as will be discussed later.

There are several studies of the Higgs-photon coupling in the MSSM [26] which however mainly focussed on the detectability of the $h \rightarrow \gamma \gamma$ signal at the LHC. In this paper, we analyze this coupling with a different perspective: we scan the entire MSSM parameter space and single out the regions where the SUSY loops could give significant contributions. Our aim is to answer to the important question [since the measurement of the Higgs-photon coupling is one of the most important goals of the presently discussed $\gamma \gamma$ colliders] of how well one needs to measure the $\Phi \rightarrow \gamma \gamma$ width in order to discriminate between the SM and the MSSM Higgs boson in the decoupling regime, if no SUSY particle has been observed directly at the LHC or at an $e^{+} e^{-}$collider with a c.m. energy of $\sqrt{s}=500 \mathrm{GeV}$.

The paper is organized as follows. In the next section, we describe the Higgs sector in the decoupling limit and present for completeness the formulae for the loop contributions to the $\Phi \gamma \gamma$ coupling. In section 3, we analyze the Higgs production at $\gamma \gamma$ colliders, and estimate the precision with which the Higgs-photon coupling can be measured. In section 4 we discuss the various contributions and isolate the parameter space in which these contributions are significant. Our conclusions will be given in section 5. In the Appendix, we derive the QCD correction to the squark loop contribution to the $\Phi \gamma \gamma$ amplitude.

\footnotetext{
${ }^{1}$ At $e^{+} e^{-}$colliders one can also measure the $e^{+} e^{-} \rightarrow \Phi \gamma$ cross section which is built up by loops of heavy particles [16]; however the cross sections are rather small, and large luminosities will be required. Another possibility is provided by the process $\gamma e^{-} \rightarrow e^{-} \Phi$ as recently discussed in 17.
} 


\section{The Higgs-Photon coupling in the MSSM}

In the MSSM, using $M_{A}$ and $\tan \beta$ as input parameters, and including the leading radiative correction which can be parameterized in terms of the quantity [2]

$$
\epsilon=\frac{3 G_{F}}{\sqrt{2} \pi^{2}} \frac{m_{t}^{4}}{\sin ^{2} \beta} \log \left(1+\frac{m_{\tilde{q}}^{2}}{m_{t}^{2}}\right)
$$

with $m_{\tilde{q}}$ the common squark mass, the $\mathrm{CP}$-even Higgs boson masses are given by

$$
M_{h, H}^{2}=\frac{1}{2}\left(M_{A}^{2}+M_{Z}^{2}+\epsilon\right)\left[1 \mp \sqrt{1-4 \frac{M_{A}^{2} M_{Z}^{2} \cos ^{2} 2 \beta+\epsilon\left(M_{A}^{2} \sin ^{2} \beta+M_{Z}^{2} \cos ^{2} \beta\right)}{\left(M_{A}^{2}+M_{Z}^{2}+\epsilon\right)^{2}}}\right]
$$

In the decoupling limit, $M_{A}^{2} \gg M_{Z}^{2}$, the Higgs masses approach the values

$$
\begin{aligned}
M_{h} \rightarrow & \sqrt{M_{Z}^{2} \cos ^{2} 2 \beta+\epsilon \sin ^{2} \beta} \\
& \times\left[1+\frac{\epsilon M_{Z}^{2} \cos ^{2} \beta}{2 M_{A}^{2}\left(M_{Z}^{2} \cos ^{2} 2 \beta+\epsilon \sin ^{2} \beta\right)}-\frac{M_{Z}^{2} \sin ^{2} 2 \beta+\epsilon \cos ^{2} \beta}{2 M_{A}^{2}}\right] \\
M_{H} \rightarrow & M_{A}\left[1+\frac{M_{Z}^{2} \sin ^{2} 2 \beta+\epsilon \cos ^{2} \beta}{2 M_{A}^{2}}\right]
\end{aligned}
$$

The $h$ and $H$ boson masses are displayed in Fig. 2 as a function of the pseudoscalar mass for several values of $\tan \beta=1.1,1.6,5$ and 50 and for $m_{\tilde{q}}=250 \mathrm{GeV}$ and $1 \mathrm{TeV}$. In the case of $h$, the decoupling limit $M_{h} \simeq M_{h}^{\max }$ is reached very quickly for large values of $\tan \beta$ [already for $M_{A} \sim 110 \mathrm{GeV}$ ] and the maximal $h$ mass is large, up to $M_{h}^{\max } \simeq 130$ $\mathrm{GeV}$. For small $\tan \beta$ values, the maximum $h$ mass is rather small for $\tan \beta=1.1$; this is due to the fact that $\cos 2 \beta$ is close to zero and $M_{h}$ is entirely generated through radiative corrections. The approach to the decoupling limit is rather slow, and for $\tan \beta=1.6$, the value $M_{h}^{\max } \simeq 80-100 \mathrm{GeV}$ is reached only for $M_{A} \simeq 500 \mathrm{GeV}$. In the decoupling limit, the heavy $\mathrm{CP}$-even Higgs particle becomes degenerate with the pseudoscalar, $M_{H} \sim M_{A}$. Similarly to $h$, this occurs very quickly for high $\tan \beta$ and slowly for low $\tan \beta$ values.

The charged Higgs boson mass is not affected by the large radiative correction eq. (1) and does not depend on $\tan \beta$, it is simply given by

$$
M_{H^{ \pm}}=M_{A}\left[1+\frac{M_{W}^{2}}{M_{A}^{2}}\right]^{1 / 2} \rightarrow M_{A} \text { for } M_{A} \gg M_{W}
$$

It is shown in Fig. 2 together with the $h / H$ masses. Finally, the mixing angle angle $\alpha$ which also receives large radiative corrections

$$
\tan 2 \alpha=\tan 2 \beta \frac{M_{A}^{2}+M_{Z}^{2}}{M_{A}^{2}-M_{Z}^{2}+\epsilon / \cos 2 \beta} ;-\frac{\pi}{2}<\alpha<0,
$$


reaches the values $\alpha \rightarrow \beta-\pi / 2$ in the decoupling limit.

The two-photon decay width of a $\mathrm{CP}$-even Higgs particle $\Phi=h, H$ can be written as [1]

$$
\Gamma(\Phi \rightarrow \gamma \gamma)=\frac{G_{F} \alpha^{2} M_{\Phi}^{3}}{128 \sqrt{2} \pi^{3}}\left|\sum_{i} A_{i}\left(\tau_{i}\right)\right|^{2}
$$

where the scaling variable $\tau_{i}$ is defined as $\tau_{i}=M_{\Phi}^{2} / 4 m_{i}^{2}$ with $m_{i}$ the mass of the loop particle. While in the SM one has only contributions from the $W$ boson and heavy fermions, in the MSSM additional contributions are provided by the charged Higgs boson, the two chargino states and the scalar partners of the fermions; Fig. 1. Factorizing the reduced couplings of these particles to the Higgs boson and to the photons, the amplitudes of the various contributions read [1]

$$
\begin{aligned}
A_{W} & =g_{\Phi W W} F_{1}\left(\tau_{W}\right) \\
A_{f} & =N_{c} Q_{f}^{2} g_{\Phi f f} F_{1 / 2}\left(\tau_{f}\right) \\
A_{H^{ \pm}} & =g_{\Phi H^{+} H^{-}} \frac{M_{W}^{2}}{M_{H^{ \pm}}^{2}} F_{0}\left(\tau_{H^{ \pm}}\right) \\
A_{\chi_{i}} & =g_{\Phi \chi_{i}^{+} \chi_{i}^{-}} \frac{M_{W}}{m_{\chi_{i}}} F_{1 / 2}\left(\tau_{\chi_{i}}\right) \\
A_{\tilde{f}_{i}} & =N_{c} Q_{f}^{2} g_{\Phi \tilde{f}_{i} \tilde{f}_{i}} \frac{M_{Z}^{2}}{m_{\tilde{f}_{i}}^{2}} F_{0}\left(\tau_{\tilde{f}_{i}}\right)
\end{aligned}
$$

with $N_{c}$ the color factor and $Q_{f}$ the electric charge of the (s)fermion in units of the proton charge. With the help of the function $f(\tau)$ defined by

$$
f(\tau)= \begin{cases}\arcsin ^{2} \sqrt{\tau} & \tau \leq 1 \\ -\frac{1}{4}\left[\log \frac{1+\sqrt{1-\tau^{-1}}}{1-\sqrt{1-\tau^{-1}}}-i \pi\right]^{2} & \tau>1\end{cases}
$$

the spin 1, 1/2 and spin 0 amplitudes are given by [1]

$$
\begin{aligned}
F_{1}(\tau) & =\left[2 \tau^{2}+3 \tau+3(2 \tau-1) f(\tau)\right] / \tau^{2} \\
F_{1 / 2}(\tau) & =-2[\tau+(\tau-1) f(\tau)] / \tau^{2} \\
F_{0}(\tau) & =[\tau-f(\tau)] / \tau^{2}
\end{aligned}
$$

The amplitudes are real if the Higgs mass is below the particle threshold, $M_{\Phi}<2 m_{i}$, while they are complex above this threshold. In the limit of heavy loop masses, $\tau \ll 1$, these amplitudes reach the asymptotic values

$$
F_{1} \rightarrow+7 \quad, \quad F_{1 / 2} \rightarrow-\frac{4}{3} \text { and } F_{0} \rightarrow-\frac{1}{3}
$$

Note that while the $W$ and fermion loops give finite contributions in the asymptotic limit, the contributions of the charged Higgs boson, the charginos and the sfermions vanish in the large loop mass limit since the amplitudes $A_{i}$ are damped by the heavy masses. 


\section{Higgs production at $\gamma \gamma$ Colliders}

The two-photon width of Higgs bosons can be directly measured at $\gamma \gamma$ colliders, with the photons generated by Compton back-scattering of laser beams from electron beams [23]. The electron and laser beam polarizations can be chosen such as to tune the photon energy spectrum and produce a peak at a fixed energy. The energy of the $\gamma \gamma$ collider can be as much as $\sim 80 \%$ of that of the original $e^{+} e^{-}$collider [19, 23. In the following, we will discuss briefly the production of the SM Higgs boson in the intermediate mass range at photon colliders; we will consider the various backgrounds and estimate the precision with which the $H^{0} \rightarrow \gamma \gamma$ width could be measured. For this purpose we will follow Ref. [19] from which the collider configuration, like beam polarization, conversion distance, etc., is taken from. We will assume that the Higgs boson mass $M_{H}$ is already known and therefore, choose the option in which the beam energy is tuned for the $\gamma \gamma$ luminosity to peak at $M_{H}$. We also adopt the option where the handedness of the electron/positron beams and laser photons are opposite in order to enhance the $J_{Z}=0$ partial wave in which the Higgs boson signal occurs.

For masses below $M_{H} \lesssim 130 \mathrm{GeV}$, the Higgs boson will dominantly decay into $b \bar{b}$ pairs; decays into charm quarks, $\tau$ leptons and gluons occur at the level of a few percent and for masses close to $M_{H} \sim 130 \mathrm{GeV}$, the $W W$ decay mode becomes important and reaches a branching ratio of $\sim 30 \%$ [27. The Higgs boson is extremely narrow, with a total decay width below $\sim 10 \mathrm{MeV}$. Being induced by loops, the $H^{0} \rightarrow \gamma \gamma$ width is very small, of order of a few $10 \mathrm{keV}$ in the mass range $80 \mathrm{GeV} \lesssim M_{H} \lesssim 130 \mathrm{GeV}$. For a complete discussion, see Ref. [27] from which we take the inputs for masses and couplings.

Since the process we are interested in proceeds through a very narrow resonance, the detector accuracy when comparing the $\gamma \gamma \rightarrow H^{0} \rightarrow b \bar{b}$ signal and the background should

be taken into account. A simple way to obtain the effective signal and backgrounds consists of introducing a gaussian smearing of the two-photon invariant mass $W$,

$$
L_{\text {eff }} \frac{\mathrm{d} \sigma^{\text {eff }}}{\mathrm{d} W}(W)=\int_{M_{X}}^{y_{m} \sqrt{s_{e^{+} e^{-}}}} \mathrm{d} W^{\prime} \frac{1}{\sqrt{2 \pi} \delta} \exp \left\{-\frac{\left(W^{\prime}-W\right)^{2}}{2 \delta^{2}}\right\} \frac{\mathrm{d} L}{\mathrm{~d} W^{\prime}} \hat{\sigma}\left(W^{\prime}\right)
$$

and selecting events within a bin of invariant masses $M_{H} \pm \Delta$. In the previous expression, $L_{\text {eff }}$ and $y_{m} \sqrt{s_{e^{+} e^{-}}}$are the effective luminosity and the maximum energy of the $\gamma \gamma$ collider; $\delta$ is one sigma of the detector resolution for $W$. The cross section for the signal process $\gamma \gamma \rightarrow H^{0} \rightarrow b \bar{b}$ can be written as

$$
\hat{\sigma}_{\mathrm{SG}}(W)=4 \pi^{2} \frac{\Gamma\left(H^{0} \rightarrow \gamma \gamma\right) \mathrm{BR}\left(H^{0} \rightarrow b \bar{b}\right)}{M_{H}^{2}}\left(1+\lambda_{1} \lambda_{2}\right) \delta\left(W-M_{H}\right),
$$

where the helicities of the scattered photons must be such that $\lambda_{1} \lambda_{2}=1$. Inserting the 
cross section in eq. (11), and selecting the events in the bin $M_{H} \pm \Delta$, one obtains

$$
L_{\text {eff }} \sigma_{\mathrm{SG}}^{\mathrm{eff}}\left(M_{H}\right)=\left.R(\Delta / \delta) \frac{\mathrm{d} L^{J_{Z}=0}}{\mathrm{~d} W}\right|_{W=M_{H}} 8 \pi^{2} \frac{\Gamma\left(H^{0} \rightarrow \gamma \gamma\right) B\left(H^{0} \rightarrow X\right)}{M_{H}^{2}},
$$

with $R(\Delta / \delta)$ being the Gaussian error function, describing the fraction of signal events contained in the bin $M_{H} \pm \Delta$ [for instance, for $\Delta=1.25 \delta$ one has $R \simeq 0.75$ ].

In the intermediate mass range, $80 \mathrm{GeV} \lesssim M_{H} \lesssim 130 \mathrm{GeV}$, the main source of background is the continuum production of $b$ - and $c$-quark pairs, including gluon radiation which leads to fake two-jet events [21]. The contribution of resolved photons to heavy quark production is rather controversial since the partonic distribution functions for polarised photons are not yet available; see the discussions given in [19, 22]. Another potentially large background for $M_{H} \sim M_{Z}$, is the process $e \gamma \rightarrow(e) Z \rightarrow b \bar{b}$ which comes from the residual electrons that were left over from the Compton scattering; the scattered electron is emitted backwards down the beampipe. It can be reduced by removing the residual electrons from the interaction region with a strong magnetic field, and this requires a non zero conversion distance 23]. Finally, we have $\gamma \gamma \rightarrow Z(f \bar{f}) \rightarrow b \bar{b}$ 19, 24 which would constitute a serious problem that may be overcome using a very peaked $\gamma \gamma$ luminosity distribution at $W \approx M_{Z}$ to greatly increase the signal-to-background ratio.

Here, we will only include the continuum $q \bar{q}$ and $q \bar{q}(g)$ backgrounds that we calculated using the package HELAS [25]. As in Ref. [20], we have used the following set of experimental cuts for the signal and backgrounds: (i) both jets from the $q$ and $\bar{q}$ should be visible in the detector: $\left|\cos \theta_{q, \bar{q}}\right|<0.7$; (ii) the gluon jet should escape detection: $\left|\cos \theta_{g}\right|>0.9$; (iii) the jets should be clearly isolated: $m_{i j}^{2} / s_{e^{+} e^{-}}>0.02$; (iv) the missing $p_{T}$ and the aplanarity due to missing gluon should be small: $\not \phi_{T}<10 \mathrm{GeV}$ and ||$\phi_{q}+\phi_{\bar{q}}|-\pi|<0.02$. For the detector accuracy, we also employ the same resolution as in Ref. [20]: $\delta=4 \mathrm{GeV}$ and $\Delta=5 \mathrm{GeV}$ for half of the width of the selection interval. The effective cross sections for a tuned energy $0.8 \sqrt{s_{e^{+} e^{-}}}=M_{H}$ are given in Table 1 for three choices of the Higgs boson mass $M_{H}=80,105$ and $130 \mathrm{GeV}$.

\begin{tabular}{|l|c|c|c|}
\hline Process & $M_{H}=80 \mathrm{GeV}$ & $M_{H}=105 \mathrm{GeV}$ & $M_{H}=130 \mathrm{GeV}$ \\
\hline \hline$\gamma \gamma \rightarrow H^{0} \rightarrow b \bar{b}$ & 67.9 & 73.0 & 62.6 \\
\hline \hline$\gamma \gamma \rightarrow b \bar{b}$ & 18.1 & 7.13 & 3.40 \\
\hline$\gamma \gamma \rightarrow c \bar{c}$ & 240 & 99.4 & 49.2 \\
\hline$\gamma \gamma \rightarrow b \bar{b}(g)$ & 0.13 & 0.05 & 0.02 \\
\hline$\gamma \gamma \rightarrow c \bar{c}(g)$ & 1.72 & 0.72 & 0.36 \\
\hline
\end{tabular}

Table 1: Effective cross sections [in fb] for the signal and the backgrounds for a tuned energy $0.8 \sqrt{s_{e^{+} e^{-}}}=M_{H}$ and the luminosity distribution of Fig. 16 of Ref. 19]. 
For the $b \bar{b}$ final state, we will assume a detection efficiency of $50 \%$ [which should be achieved in the future by micro-vertex detectors] with a $5 \%$ contamination from $c \bar{c}$ final states. One the other hand, only the events where both quarks decay hadronically should be collected to estimate the $\gamma \gamma$ invariant mass; the hadronic decay branching ratios of $b-$ and $c$-flavored hadrons are $75 \%$ and $82 \%$ respectively. Multiplying the tagging efficiencies times the square of the hadronic branching ratios, we obtain the corrected effective cross sections in Table 2. As can be seen, the radiative background is completely negligible and the signal cross sections are much larger than the backgrounds, especially for high Higgs boson masses, leading to a large statistical significance for the Higgs boson signal.

\begin{tabular}{|l|c|c|c|}
\hline Process & $M_{H}=80 \mathrm{GeV}$ & $M_{H}=105 \mathrm{GeV}$ & $M_{H}=130 \mathrm{GeV}$ \\
\hline \hline$\gamma \gamma \rightarrow H^{0} \rightarrow b \bar{b}$ & 19.1 & 20.5 & 17.6 \\
\hline \hline$\gamma \gamma \rightarrow b \bar{b}$ & 5.09 & 2.00 & 0.96 \\
\hline$\gamma \gamma \rightarrow c \bar{c}$ & 8.07 & 3.34 & 1.65 \\
\hline$\gamma \gamma \rightarrow b \bar{b}(g)$ & 0.037 & 0.014 & 0.006 \\
\hline$\gamma \gamma \rightarrow c \bar{c}(g)$ & 0.058 & 0.024 & 0.012 \\
\hline \hline Total Background & 13.3 & 5.38 & 2.63 \\
\hline \hline Signal/Background & 1.44 & 3.81 & 6.69 \\
\hline Stat. Significance & 16.6 & 27.9 & 34.3 \\
\hline Sensitivity to $\Gamma_{\gamma \gamma}$ & $9.4 \%$ & $7.8 \%$ & $8.1 \%$ \\
\hline
\end{tabular}

Table 2: Corrected effective cross sections [in fb] to include tagging efficiency and the invariant mass reconstruction. An integrated luminosity of $10 \mathrm{fb}^{-1}$ is assumed for the evaluation of the statistical significance and the sensitivity to the two-photon width of the Higgs boson.

The measurement of $\Gamma\left(H^{0} \rightarrow \gamma \gamma\right) \times \mathrm{BR}\left(H^{0} \rightarrow \gamma \gamma\right)$ will follow from eq. (13) if the luminosity, the tagging and mass reconstruction efficiencies as well as the Higgs boson mass are precisely known. Assuming that $\mathrm{BR}\left(H^{0} \rightarrow \gamma \gamma\right)$ is given by the $\mathrm{SM}$ and that the uncertainties in all the previous quantities are negligible, the statistical error in the $\Gamma\left(H^{0} \rightarrow \gamma \gamma\right)$ determination is

$$
\frac{\Delta \Gamma}{\Gamma}=\frac{1}{\sqrt{L_{\mathrm{eff}}}} \frac{\sqrt{S+B}}{S}
$$

which is about $10 \%$ for an effective luminosity $L_{\mathrm{eff}}=10 \mathrm{fb}^{-1}$. Increasing the luminosity and improving the $b$-tagging efficiency and purity as well as the reconstruction of the $b \bar{b}$ invariant mass would enhance the sensitivity to the $h \gamma \gamma$ coupling. 


\section{Loop contributions in the MSSM}

\section{1 $W$ boson loop}

Compared to the SM case where $g_{H^{0} W W}=1$, the $W$ boson amplitude for the lightest MSSM Higgs particle $h$ is suppressed by a factor $g_{h W W}=\sin (\beta-\alpha)$. However, in the decoupling regime $M_{A}^{2} \gg M_{Z}^{2}$, the $h W W$ coupling approaches quickly the SM coupling

$$
g_{h W W}=\sin (\beta-\alpha) \rightarrow 1-\frac{1}{8} \sin ^{2} 4 \beta \frac{M_{Z}^{4}}{M_{A}^{4}}\left[1-\frac{\epsilon}{2 M_{Z}^{2} \cos 2 \beta}\right]^{2} \rightarrow 1
$$

The $W$ boson form factor $A_{W}$ is shown in Fig. 3a as a function of $M_{h}$ both in the SM and in the MSSM. The MSSM contribution has been obtained by fixing the pseudoscalar mass to $M_{A}=250 \mathrm{GeV}$ and varying the value of $\tan \beta$ from $\tan \beta=1.1$ to 50 . The difference between the SM and the MSSM contributions is very small, even for low $\tan \beta$ values where the decoupling limit is not completely reached yet for $M_{A}=250 \mathrm{GeV}$. This is due to the fact that $\sin (\beta-\alpha)$ approaches unity very quickly, the difference being of $\mathcal{O}\left(M_{Z}^{4} / M_{A}^{4}\right)$.

\subsection{Fermion loops}

Since the $\Phi f f$ couplings are proportional to the fermion mass, the contribution of the light fermions to the $\Phi \gamma \gamma$ amplitude is negligible. Only the top quark, and to a smaller extent the charm and bottom quark, as well as the $\tau$ lepton, will effectively contribute. Compared to the SM case where $g_{H^{0} f f}=1$, the $h u u / h d d$ couplings are suppressed/enhanced by the factors

$$
\begin{aligned}
& g_{\text {huu }}=\frac{\cos \alpha}{\sin \beta} \rightarrow 1+\frac{1}{2} \frac{M_{Z}^{2}}{M_{A}^{2}} \cot \beta \sin 4 \beta\left[1-\frac{\epsilon}{2 M_{Z}^{2} \cos 2 \beta}\right] \rightarrow 1 \\
& g_{\text {hdd }}=-\frac{\sin \alpha}{\cos \beta} \rightarrow 1-\frac{1}{2} \frac{M_{Z}^{2}}{M_{A}^{2}} \tan \beta \sin 4 \beta\left[1-\frac{\epsilon}{2 M_{Z}^{2} \cos 2 \beta}\right] \rightarrow 1
\end{aligned}
$$

The fermionic amplitudes $A_{t}$ and $A_{b, c, \tau}$ are shown in Fig. $3 \mathrm{~b}$ as a function of $M_{h}$, with $M_{A}$ again fixed to $250 \mathrm{GeV}$. In the SM, the dominant fermionic contribution $A_{t}$ is almost constant and can be approximated by $A_{t} \sim N_{c} Q_{t}^{2} F_{1 / 2}(0)=-16 / 9$. It is smaller than the $W$ boson contribution and the two amplitudes interfere destructively. In the MSSM, the variation with $M_{h}$ is rather pronounced. This is due to the variation of the coupling $g_{h u u}$ since the decoupling limit is not reached yet for $M_{A}=250 \mathrm{GeV}$ and small $\tan \beta$ values: contrary to $g_{h W W}$, the coupling $g_{h u u}$ approaches the decoupling limit slowly, $g_{h u u} \rightarrow$ $1-\mathcal{O}\left(M_{Z}^{2} / M_{A}^{2}\right)$.

For the bottom quark loop, the amplitude $A_{b}$ has both real and imaginary parts since $M_{h}>2 m_{b}$. The real part of $A_{b}$, calculated with a running mass $m_{b}\left(M_{\Phi}^{2}\right) \sim 3 \mathrm{GeV}$, 
is much smaller compared to $A_{t}$ as expected[2], but is of the same order as $A_{\tau}$ since the latter is not penalized by the charge factor. The imaginary parts $\operatorname{Im}\left(A_{b, \tau}\right)$ are larger than $\operatorname{Re}\left(A_{b, \tau}\right)$, but since they do not interfere with the dominant $A_{W}$ and $A_{t}$ contributions, their effect on the $\Phi \rightarrow \gamma \gamma$ width is rather small. Note that the difference between the $\mathrm{SM}$ and MSSM is still rather large for $M_{A}=250 \mathrm{GeV}$, however, this difference will hardly be noticed in $\Gamma(h \rightarrow \gamma \gamma)$ since the contributions of the $b$ and $\tau$ loops are small.

Finally, we note that the QCD corrections to the dominant top quark loop are well under control and can be included by simply multiplying the Born amplitude by a factor $\left(1-\alpha_{s} / \pi\right)$. The QCD corrections to the $b$ quark loop do not exceed the level of a few times $\alpha_{s} / \pi$ if the running $b$ quark mass at a scale $M_{\Phi} / 2$ is used in the Born amplitude; for more details on these corrections, see Ref. [12].

\subsection{Charged Higgs boson loops}

In the coupling of the lightest $\mathrm{CP}$-even Higgs particle $h$ to charged Higgs bosons, large radiative corrections which cannot be mapped into the mixing angle $\alpha$ will appear. Retaining again only the leading correction, the $g_{h H^{+} H^{-}}$coupling is given by 28]

$$
g_{h H^{+} H^{+}}=\sin (\beta-\alpha)+\frac{\cos 2 \beta \sin (\beta+\alpha)}{2 c_{W}^{2}}+\frac{\epsilon}{2 c_{W}^{2} M_{Z}^{2}} \frac{\cos \alpha \cos ^{2} \beta}{\sin \beta}
$$

with $s_{W}^{2}=1-c_{W}^{2} \equiv \sin ^{2} \theta_{W}$. In the decoupling limit, the coupling reduces up to $\mathcal{O}(\epsilon)$ terms, to $g_{h H^{+} H^{+}} \rightarrow 1-\cos ^{2} 2 \beta /\left(2 c_{W}^{2}\right)$.

The form factor $A_{H^{ \pm}}$is shown in Fig. 4 a as a function of $M_{H^{ \pm}}$for $\tan \beta=1.6,5$ and 50. Because the contribution is damped by a factor $1 / M_{H^{ \pm}}^{2}$ for large $H^{ \pm}$masses, and also because the spin-zero amplitude $F_{0}$ is small, the charged Higgs contribution to the $h \gamma \gamma$ coupling is very small. For low masses, $M_{H^{ \pm}} \sim 100 \mathrm{GeV}, A_{H^{ \pm}}$can reach values close to $\sim-0.1$, but for $M_{H^{ \pm}} \gtrsim 250 \mathrm{GeV}$ the contribution of the $H^{ \pm}$loop is already only a few per mille of that of the dominant $W$ boson loop, and is therefore completely negligible .

\subsection{Scalar lepton and quark loops}

The left- and right-handed scalar partners of each SM charged fermion, $\tilde{f}_{L}$ and $\tilde{f}_{R}$, mix to give the mass eigenstates $\tilde{f}_{1}$ and $\tilde{f}_{2}$. The mixing angle is proportional to the fermion mass and is therefore important only in the case of the top squarks [29]; for the scalar

\footnotetext{
${ }^{2}$ In the MSSM, however, far from the decoupling limit and for large values of $\tan \beta$, the amplitude $A_{b}$ can be very large since the coupling $g_{h b b} \sim \tan \beta$ is strongly enhanced.

${ }^{3}$ Note that in two-Higgs doublet models, charged Higgs boson loops will provide the only additional contribution to the $h \gamma \gamma$ coupling. Since this contribution is very small, discriminating between this model and the SM in the decoupling regime using the $h \gamma \gamma$ coupling will not be possible.
} 
partners of light fermions the current eigenstates are identical to the mass eigenstatesf In this subsection, we will discuss the contribution of slepton and the scalar partners of the light quarks only, the contributions of top squark loops will be discussed separately later.

The reduced couplings of the $h$ boson to the left-handed and right-handed partners of light fermions, are given by

$$
\begin{aligned}
& g_{h \tilde{f}_{L} \tilde{f}_{L}}=\left(I_{3}^{f}-Q_{f} s_{W}^{2}\right) \sin (\beta+\alpha) \\
& g_{h \tilde{f}_{R} \tilde{f}_{R}}=Q_{f} s_{W}^{2} \sin (\beta+\alpha)
\end{aligned}
$$

with $I_{3}^{f}= \pm 1 / 2$ and $Q_{f}$ the weak isospin and the electric charge of the fermion $f$. In the decoupling limit, one has $\sin (\beta+\alpha) \rightarrow-\cos 2 \beta$.

The contributions of the slepton and squark [except for top squark] loops are shown in Fig. 4b as functions of the masses and for the three values $\tan \beta=1.6,5$ and 50 with $M_{A}$ fixed to $250 \mathrm{GeV}$. We have summed over all slepton and squark [except stop] contributions, and used common masses $m_{\tilde{l}}$ and $m_{\tilde{q}}$, which is approximately the case in SUSY-GUT models. The form factor $A_{\tilde{l}}$ is approximately equal to $A_{H^{ \pm}}$except that the trend for various $\tan \beta$ values is reversed. The contribution of the squark loops $A_{\tilde{q}}$ has almost the same magnitude as the contribution $A_{\tilde{l}}$, but is of opposite sign. As in the case of the charged Higgs boson, slepton and squark loop contributions to the $h \rightarrow \gamma \gamma$ decay width are very small: in the decoupling limit and for loop masses above $250 \mathrm{GeV}$, they do not exceed a few per mille and can be safely neglected.

\subsection{Top squark loops}

Due to the large value of the top quark mass, the mixing between the left- and righthanded scalar partners of the top quark, $\tilde{t}_{L}$ and $\tilde{t}_{R}$, can be very large. The mass eigenstates $\tilde{t}_{1}$ and $\tilde{t}_{2}$ are obtained by diagonalizing the mass matrix

$$
\mathcal{M}_{\tilde{t}}^{2}=\left(\begin{array}{cc}
m_{\tilde{t}_{L}}^{2}+m_{t}^{2}+\cos 2 \beta\left(\frac{1}{2}-\frac{2}{3} s_{W}^{2}\right) M_{Z}^{2} & m_{t} m_{t}^{L R} \\
m_{t} m_{t}^{L R} & m_{\tilde{t}_{R}}^{2}+m_{t}^{2}+\frac{2}{3} \cos 2 \beta s_{W}^{2} M_{Z}^{2}
\end{array}\right)
$$

where the left- and right-handed scalar masses $m_{\tilde{t}_{L}}$ and $m_{\tilde{t}_{R}}$ are generally assumed to be approximately equal to the common mass of the scalar partners of light quarks $m_{\tilde{q}}$. In terms of the soft-SUSY breaking trilinear couplings $A_{t}$ and the Higgs-higgsino mass parameter $\mu$, the off-diagonal term $m_{t}^{L R}$ reads

$$
m_{t}^{L R}=A_{t}-\mu \cot \beta
$$

\footnotetext{
${ }^{4}$ The mixing in the sbottom sector can also be sizeable for large values of $\tan \beta$. We have checked explicitely that this mixing will not affect significantly the numerical results compared to the no-mixing case, if the value of the off-diagonal entry in the sbottom mass matrix is not prohibitively large.
} 
The top squark masses and the mixing angle are then given by

$$
\begin{gathered}
m_{\tilde{t}_{1}, \tilde{t}_{2}}^{2}=m_{t}^{2}+\frac{1}{2}\left[m_{\tilde{t}_{L}}^{2}+m_{\tilde{t}_{R}}^{2} \mp \sqrt{\left(m_{\tilde{t}_{L}}^{2}-m_{\tilde{t}_{R}}^{2}\right)^{2}+\left(2 m_{t} m_{t}^{L R}\right)^{2}}\right] \\
\sin 2 \theta_{t}=\frac{2 m_{t} m_{t}^{L R}}{m_{\tilde{t}_{1}}^{2}-m_{\tilde{t}_{2}}^{2}} \quad, \quad \cos 2 \theta_{t}=\frac{m_{\tilde{t}_{L}}^{2}-m_{\tilde{t}_{R}}^{2}}{m_{\tilde{t}_{1}}^{2}-m_{\tilde{t}_{2}}^{2}}
\end{gathered}
$$

The couplings of the $h$ boson to top squarks in the presence of mixing are given by

$$
\begin{aligned}
g_{h \tilde{t}_{1} \tilde{t}_{1}}= & \frac{1}{2} \sin (\alpha+\beta)\left[\cos ^{2} \theta_{t}-\frac{4}{3} s_{W}^{2} \cos 2 \theta_{t}\right]-\frac{\cos \alpha}{\sin \beta} \frac{m_{t}^{2}}{M_{Z}^{2}} \\
& +\frac{m_{t} \sin 2 \theta_{t}}{2 M_{Z}^{2}}\left[\frac{\cos \alpha}{\sin \beta} A_{t}+\frac{\sin \alpha}{\sin \beta} \mu\right] \\
g_{h \tilde{t}_{2} \tilde{t}_{2}}= & \frac{1}{2} \frac{\sin (\alpha+\beta)}{2}\left[\sin ^{2} \theta_{t}+\frac{4}{3} s_{W}^{2} \cos 2 \theta_{t}\right]-\frac{\cos \alpha}{\sin \beta} \frac{m_{t}^{2}}{M_{Z}^{2}} \\
& -\frac{m_{t} \sin 2 \theta_{t}}{2 M_{Z}^{2}}\left[\frac{\cos \alpha}{\sin \beta} A_{t}+\frac{\sin \alpha}{\sin \beta} \mu\right]
\end{aligned}
$$

In the decoupling limit, these vertices reduce to

$$
\begin{aligned}
& g_{h \tilde{t}_{1} \tilde{t}_{1}}=-\frac{1}{2} \cos 2 \beta\left[\cos ^{2} \theta_{t}-\frac{4}{3} s_{W}^{2} \cos 2 \theta_{t}\right]-\frac{m_{t}^{2}}{M_{Z}^{2}}+\frac{1}{2} \sin 2 \theta_{t} \frac{m_{t} m_{t}^{L R}}{M_{Z}^{2}} \\
& g_{h \tilde{t}_{2} \tilde{t}_{2}}=-\frac{1}{2} \cos 2 \beta\left[\sin ^{2} \theta_{t}+\frac{4}{3} s_{W}^{2} \cos 2 \theta_{t}\right]-\frac{m_{t}^{2}}{M_{Z}^{2}}-\frac{1}{2} \sin 2 \theta_{t} \frac{m_{t} m_{t}^{L R}}{M_{Z}^{2}}
\end{aligned}
$$

Assuming as usual that $m_{\tilde{t}_{L}}=m_{\tilde{t}_{R}}=m_{\tilde{q}}$, the only parameters which enter the contribution of the $\tilde{t}$ loops to the $h \rightarrow \gamma \gamma$ decay width in the decoupling limit are $m_{\tilde{q}}$ [that we will trade against $\left.m_{\tilde{t}_{1}}\right]$ and $m_{t}^{L R}$. There is also a dependence on $\tan \beta$ which arises from the Higgs coupling to top squarks and from the mixing angle since the stop mass matrix contains also a small $\cos 2 \beta$ term. However, this dependence on $\tan \beta$ is rather small.

In Fig. 5 a, we show contour plots in the $\left(m_{t}^{L R}, m_{\tilde{t}_{1}}\right)$ plane for which the contribution $A_{\tilde{t}}$ [which includes the amplitudes of both top squarks] to the $h \gamma \gamma$ coupling is $\left|A_{\tilde{t}}\right|=2,1,0.5$ and 0.2. For the sake of convenience, we also display in Fig. 5b, contours in the $\left(m_{t}^{L R}, m_{\tilde{t}_{1}}\right)$ plane for fixed masses of the heavy top squark and the scalar partners of the light squarks. To have a better insight on the various contributions, we show in Fig. 6 three dimensional plots of the two separate top squark amplitudes $A_{\tilde{t}_{1}}$ and $A_{\tilde{t}_{2}}$.

The amplitude $A_{\tilde{t}}$ is symmetric for positive and negative $m_{t}^{L R}$ values because for large $m_{t}^{L R}$, the $h \tilde{t} \tilde{t}$ coupling is dominated by the $\sin 2 \theta_{t} m_{t}^{L R}$ term and $\sin 2 \theta_{t}$ is proportional to $m_{t}^{L R}$; for small $m_{t}^{L R}$ the dominant piece of the $h \tilde{t} \tilde{t}$ coupling is proportional to $m_{t}^{2}$. To discuss the effect of the mixing, it is convenient to divide the parameter space into three 
regions: intermediate $\left|m_{t}^{L R}\right|$ values around the region delimited by the contour $A_{\tilde{t}}=0$, large and small $\left|m_{t}^{L R}\right|$ values away from this contour.

For large $\left|m_{t}^{L R}\right|$, the contributions are large and positive; for light enough top squarks, $m_{\tilde{t}_{1}} \sim 100 \mathrm{GeV}$, they can reach the value $A_{\tilde{t}} \sim 2$ for $\left|m_{t}^{L R}\right| \sim 1 \mathrm{TeV}$, therefore almost canceling the top quark loop contribution $A_{t}$. For a given $m_{\tilde{t}_{1}}, A_{\tilde{t}}$ is larger for higher values of $m_{t}^{L R}$ because in this case, the coupling $h \tilde{t} \tilde{t} \sim m_{t}^{L R}$ is strongly enhanced. For large $m_{\tilde{t}_{1}}$, the two top squarks will have comparable masses [see Fig. 5b], and since the signs in the dominant component of the $h \tilde{t}_{1} \tilde{t}_{1}$ and $h \tilde{t}_{2} \tilde{t}_{2}$ couplings are opposite, the two amplitudes will partly cancel each other; Fig. 6.

For small $\left|m_{t}^{L R}\right|$, there is a region [the small "menhir" around $m_{t}^{L R}=0$ ] where no solution for $m_{\tilde{t}_{1}}<m_{t}$ is allowed when diagonalizing the mass matrix; however, this region is already excluded by $\mathrm{CDF} / \mathrm{D} 0$ data from the negative search of scalar partners of light quarks with masses $m_{\tilde{q}} \lesssim 150 \mathrm{GeV}$ [30]; Fig. 5b. The amplitudes in this region are negative since the dominant component of the $h \tilde{t} \tilde{t}$ is now proportional to $m_{t}^{2}$ and has opposite sign compared to the dominant off diagonal coupling when $m_{t}^{L R}$ is large. $A_{\tilde{t}}$ decreases with increasing top squark mass as expected, and can reach the almost maximal value $A_{\tilde{t}} \sim-0.5$ for $m_{\tilde{t}_{1}} \lesssim 250 \mathrm{GeV}$; however, most of this region is again ruled out by the CDF/D0 bound $m_{\tilde{q}} \gtrsim 150 \mathrm{GeV}$ as shown in Fig. $5 \mathrm{~b}$.

For intermediate values of $\left|m_{t}^{L R}\right|$, there is a balance between the two components of the $h \tilde{t}_{1} \tilde{t}_{1}$ coupling which tend to cancel each other, and the two contributions $A_{\tilde{t}_{1}}$ and $A_{\tilde{t}_{2}}$ which become comparable [since for relatively large $m_{\tilde{t}_{1}}, \tilde{t}_{1}$ and $\tilde{t}_{2}$ have comparable masses, Fig. 5b, and $A_{\tilde{t}_{1}}$ is small] and interfere destructively. At some stage, the two contributions cancel each other leading to the contour $A_{\tilde{t}}=0$ of Fig. 5a. As one can see, for top squarks not much heavier than $\sim 250 \mathrm{GeV}$, one can have contributions of the order of $10 \%$ or more to the $h \rightarrow \gamma \gamma$ decay width if the off-diagonal entry in the stop mass matrix is large, $\left|m_{t}^{L R}\right| \gtrsim 1 \mathrm{TeV}$.

Since top squark contributions can be relatively large, we have derived the two-loop QCD corrections to the scalar quark loops in the limit of heavy squarks, using low energy theorems. The derivation of the result is done in the Appendix. The effect of the QCD corrections is to shift the value of the Born form-factor $A_{\tilde{q}}$ by an amount

$$
A_{\tilde{q}}=A_{\tilde{q}}^{\text {Born }}\left[1+\frac{8}{3} \frac{\alpha_{s}}{\pi}\right]
$$

The correction is about three times larger than in the case of quark loops, and has opposite sign. It is of the order of $\sim 10 \%$, and therefore well under control. 


\subsection{Chargino loops}

The masses of the two chargino states depend on $\tan \beta$, the gaugino mass parameter $M_{2}$ and the Higgs-higgsino mass parameter $\mu$ :

$$
\begin{aligned}
m_{\chi_{1,2}}^{2}= & \frac{1}{2}\left[M_{2}^{2}+\mu^{2}+2 M_{W}^{2}\right. \\
& \left.\mp \sqrt{\left(M_{2}^{2}-\mu^{2}\right)^{2}+4 M_{W}^{4} \cos ^{2} 2 \beta+4 M_{W}^{2}\left(M_{2}^{2}+\mu^{2}+2 M_{2} \mu \sin 2 \beta\right.}\right]
\end{aligned}
$$

The chargino couplings to the lightest Higgs boson $h$ are given by 31

$$
\begin{aligned}
& g_{h \chi_{1}^{+} \chi_{1}^{-}}=\sqrt{2}\left[\cos \alpha \cos \theta^{+} \sin \theta^{-}+\sin \alpha \sin \theta^{+} \cos \theta^{-}\right] \\
& g_{h \chi_{2}^{+} \chi_{2}^{-}}=-\varepsilon \sqrt{2}\left[\cos \alpha \cos \theta^{-} \sin \theta^{+}+\sin \alpha \sin \theta^{-} \cos \theta^{+}\right]
\end{aligned}
$$

with $\varepsilon=\operatorname{sign}\left(M_{2} \mu-M_{W}^{2} \sin 2 \beta\right)$ and the angles $\theta^{ \pm}$according to

$$
\begin{aligned}
\tan 2 \theta^{-} & =\frac{2 \sqrt{2} M_{W}\left(M_{2} \cos \beta+\mu \sin \beta\right)}{M_{2}^{2}-\mu^{2}-2 M_{W}^{2} \cos 2 \beta} \\
\tan 2 \theta^{+} & =\frac{2 \sqrt{2} M_{W}\left(M_{2} \sin \beta+\mu \cos \beta\right)}{M_{2}^{2}-\mu^{2}+2 M_{W}^{2} \cos 2 \beta}
\end{aligned}
$$

Note that in the decoupling limit, one has $\cos \alpha=\sin \beta$ and $\sin \alpha=-\cos \beta$.

The contribution $A_{\chi}$ of the chargino loops to the $h \gamma \gamma$ coupling is shown in Fig. 7 in the $\left(M_{2}, \mu\right)$ plane for the two values $\tan \beta=1.6$ and 50 . Contours for $\left|A_{\chi}\right|=0.5,0.3,0.2$ and 0.1 as well as the region of the parameter space for which the lightest chargino mass is larger than $90 \mathrm{GeV}$ [a value below which the charginos will be found at LEP2] and 250 $\mathrm{GeV}$ [which will be probed at a $500 \mathrm{GeV} e^{+} e^{-}$collider], have been drawn.

The chargino contributions to the $h \gamma \gamma$ coupling are much larger than those of charged Higgs bosons, sleptons and the scalar partners of light quarks. This is due to the fact that for heavy particles, the amplitude $F_{1 / 2} \rightarrow-4 / 3$ is larger than $F_{0} \rightarrow-1 / 3$, and also because the chargino contribution scales like $A_{\chi} \sim 1 / m_{\chi}$ contrary to the amplitudes for scalars which scale like $A_{i} \sim 1 / m_{i}^{2}$. For high $\tan \beta$ the contributions are positive, while for low $\tan \beta$ the amplitudes follow the sign of $\mu$.

The largest contributions are obtained for small values of $\tan \beta$. For chargino masses very close to the LEP2 limit, $m_{\chi} \sim 100 \mathrm{GeV}$, one can have to $A_{\chi} \gtrsim 0.5$ inducing contributions to $\Gamma(h \rightarrow \gamma \gamma)$ which exceed the $10 \%$ level. For chargino masses above $250 \mathrm{GeV}$, the maximum $A_{\chi}$ contribution will be below $\sim 0.2$ for both values of $\tan \beta$, altering the total $h \rightarrow \gamma \gamma$ width by less than $10 \%$ percent. 


\section{Conclusions}

We have analyzed the contribution of charged Higgs bosons, charginos, sleptons and squark loops to the coupling of the lightest neutral Higgs boson to two photons in supersymmetric theories. Our aim was to determine the region of the MSSM parameter space in which one is still sensitive to the additional SUSY loops, although no SUSY particle has been produced directly at the LHC or at a future $e^{+} e^{-}$collider with a c.m. energy of $\sqrt{s}=500 \mathrm{GeV}$. We focussed on the decoupling limit where all the additional MSSM Higgs bosons are very heavy. In this limit, the $h$ boson will have practically the same properties as the standard Higgs particle, and the two-photon decay could be used to discriminate between the SM and the MSSM Higgs sectors. Our conclusions are as follows:

The contributions of charged Higgs bosons, sleptons and the scalar partners of the light quarks including the bottom squarks are extremely small. This is due to the fact that these particles do not couple to the Higgs boson proportionally to the mass, and the amplitude is damped by inverse powers of the heavy mass squared; in addition, the scalar loop amplitude is much smaller than the dominant $W$ amplitude. For masses above 250 $\mathrm{GeV}$, the effect of scalar particles [with the exception of the top squark] on the $h \rightarrow \gamma \gamma$ width does not exceed one precent level and can therefore be neglected.

The contribution of the charginos to the two-photon decay width can exceed the $10 \%$ level for masses close to $m_{\chi} \sim 100 \mathrm{GeV}$, but it becomes smaller with higher masses. The deviation of the $\Gamma(h \rightarrow \gamma \gamma)$ width from the SM value induced by charginos with masses $m_{\chi}=250$ and $400 \mathrm{GeV}$ is shown in Fig. 8a, as a function of $M_{2}$ [ $\mu$ is fixed by $m_{\chi}$ ] for $\tan \beta=1.6$ and 50. For chargino masses above $m_{\chi} \gtrsim 250 \mathrm{GeV}$ [i.e. slightly above the limit where charginos can be produced at a $500 \mathrm{GeV} e^{+} e^{-}$collider], the deviation is less than $\sim 8 \%$ for the entire SUSY parameter space. The deviation drops by a factor of two if the chargino mass is increased to $400 \mathrm{GeV}$.

Because its coupling to the lightest Higgs boson can be strongly enhanced, the top squark can generate sizeable contributions to the two-photon decay width. For stop masses in the $\sim 100 \mathrm{GeV}$ range, the contribution could reach the level of the dominant $W$ boson contribution and the interference is constructive increasing drastically the decay width. For $\tilde{t}_{1}$ masses around $250 \mathrm{GeV}$, the deviation of the $h \rightarrow \gamma \gamma$ decay width from the SM value can be still at the level of $10 \%$ for a very large off-diagonal entry in the stop mass matrix, $m_{t}^{L R} \gtrsim 1 \mathrm{TeV}$ [Fig. 8b]. For larger masses, the deviation drops $\sim 1 / m_{\tilde{t}_{1}}^{2}$ and the effect on the decay width is below $2 \%$ for $m_{\tilde{t}_{1}} \sim 400 \mathrm{GeV}$ even at $m_{t}^{L R} \sim 1 \mathrm{TeV}$.

\footnotetext{
${ }^{5}$ The maximum deviation is obtained for $M_{2}$ values slightly above the lightest chargino mass. The reason is that the Higgs boson prefers to couple to mixtures of gauginos and higgsinos, and in this region the $h \chi_{1}^{+} \chi_{1}^{-}$coupling is maximal. For larger $M_{2}$ values, $\chi_{1}^{+}$is a pure higgsino while for smaller $M_{2}$ values it is a pure gaugino and the Higgs coupling is therefore small.
} 
For small values of $m_{t}^{L R}$, the deviation does not exceed $-8 \%$ even for a light top squark $m_{\tilde{t}_{1}} \sim 250 \mathrm{GeV}$. For this stop mass value, we have cut out the region $m_{t}^{L R} \lesssim 200 \mathrm{GeV}$ since there, the scalar partners of light quarks will have masses smaller than $m_{\tilde{q}} \sim 250$ $\mathrm{GeV}$.

Thus, the only way to have a contribution to the two-photon decay width of the $h$ boson that is larger than $10 \%$ is from a rather light stop squark $m_{\tilde{t}_{1}} \lesssim 300 \mathrm{GeV}$ with extremely large couplings to the Higgs boson, $m_{t}^{L R}>1 \mathrm{TeV}$. However, for $m_{\tilde{t}_{1}} \sim 300$ $\mathrm{GeV}$ and $m_{t}^{L R} \lesssim 1 \mathrm{TeV}$, the scalar partners of the light quarks will have masses around $m_{\tilde{q}} \sim 500 \mathrm{GeV}$ and therefore should be observed at the LHC. The only way to have a light top squark, $m_{\tilde{t}_{1}} \sim 300 \mathrm{GeV}$, while the other squarks are heavier than $1 \mathrm{TeV}$ and

escape detection at the LHC is to increase $m_{t}^{L R}$ to $\sim 5 \mathrm{TeV}$. For such large $m_{t}^{L R}$ values, the trilinear scalar interaction become extremely strong and could lead to dynamically favoured minima of the scalar potential where charge is not conserved [32]. [A necessary, though not sufficient condition to avoid these false vacua is to choose $m_{t}^{L R} \lesssim 3 m_{\tilde{q}}$; see Ref. [32].] Furthermore, the $h \tilde{t} \tilde{t}$ coupling, $g_{h \tilde{t} \tilde{t}} \sim m_{t} m_{L R}^{2} /\left(2 M_{Z}^{2}\right)$, becomes very large and perturbation theory is endangered.

In summary: only chargino and top squark loops can lead to a sizable difference between the two-photon decay width of the lightest SUSY and the standard Higgs bosons in the decoupling limit. Charginos with masses above the production threshold of a 500 $\mathrm{GeV} e^{+} e^{-}$collider will induce contributions which are smaller than a few percent in the entire SUSY parameter space. Top squarks can induce contributions which exceed the $10 \%$ level only if they are just slightly heavier than $\sim 250 \mathrm{GeV}$ and if the off-diagonal entry in the stop mass matrix is very large, $m_{t}^{L R} \gtrsim 1 \mathrm{TeV}$. In the region of parameter space where both charginos and top squarks are light, the sum of the two contributions can exceed the $10 \%$ level.

One therefore needs a measurement of the Higgs coupling to two photons at $\gamma \gamma$ colliders with an accuracy better than $10 \%$ to discriminate between the standard and the minimal SUSY Higgs scenarios if this discrimination could not be achieved in the $e^{+} e^{-}$option of the collider with a c.m. energy in the $500 \mathrm{GeV}$ range, or if scalar quarks have not been observed at the LHC.

\section{Acknowledgments:}

Discussions with Manuel Drees, Howard Haber, Christoph Jünger, Janusz Rosiek and Michael Spira are gratefully acknowledged. J.I. is supported by a fellowship from the Fundación Ramón Areces and partially by the Spanish CICYT under contract AEN961672. 


\section{Appendix: QCD corrections to scalar quark loops}

The calculation of the QCD corrections to scalar quark loop contributions to the Higgstwo photon coupling can be calculated by extending low-energy theorems 10 to scalars at the two-loop level [12, 33]. For a CP-even Higgs boson $H$ with a mass, $M_{H} \ll 2 m_{\tilde{Q}}$ which is the case here, these theorems relate the matrix elements of the squark contributions to the $H \gamma \gamma$ vertex to the photon two-point function. In this Appendix, we will use these theorems to derive the QCD corrections to the scalar loops due to pure gluon exchange; this part of the correction is expected to be the dominant one.

Denoting the matrix element of one squark contribution to the photon self-energy by $\mathcal{M}_{\tilde{Q}}(\gamma \gamma)$, and the corresponding matrix element with an additional light Higgs boson by $\mathcal{M}_{\tilde{Q}}(\gamma \gamma H)$, one has at lowest order

$$
\mathcal{M}_{\tilde{Q}}^{\gamma \gamma H}=\left(\sqrt{2} G_{F}\right)^{1 / 2} e_{\tilde{Q}}^{2} g_{\tilde{Q}}^{H} m_{\tilde{Q}} \partial \mathcal{M}_{\tilde{Q}}^{\gamma \gamma} / \partial m_{\tilde{Q}}
$$

with $e_{\tilde{Q}}$ and $g_{\tilde{Q}}^{H}$ the squark charge and its reduced coupling to the Higgs boson. To extend this relation to higher orders, one has to replace all quantities by their bare values, differentiate with respect to $m_{\tilde{Q}}$ and then perform the renormalization. In the case of pure gluon exchange, the differentiation with respect to the bare squark mass $m_{\tilde{Q}}^{0}$ can be rewritten in terms of the renormalized mass $m_{\tilde{Q}}$. A finite contribution to the QCD corrections arises from the anomalous mass dimension $\gamma_{\tilde{Q}}$

$$
m_{\tilde{Q}}^{0} \frac{\partial}{\partial m_{\tilde{Q}}^{0}}=\frac{m_{\tilde{Q}}}{1+\gamma_{\tilde{Q}}} \frac{\partial}{\partial m_{\tilde{Q}}}
$$

The remaining differentiation with respect to the renormalized squark mass of the photon two-point function leads to the squark contribution to the $\beta$ function, $\beta_{\tilde{Q}}$. The final result for the squark contribution to the Higgs-two-photon coupling can be expressed in terms of the effective Lagrangian

$$
\mathcal{L}_{\text {eff }}^{\tilde{Q}}=\left(\sqrt{2} G_{F}\right)^{1 / 2} e_{\tilde{Q}}^{2} \frac{g_{\tilde{Q}}^{H}}{4} \frac{\beta_{\tilde{Q}} / \alpha}{1+\gamma_{\tilde{Q}}} F^{\mu \nu} F_{\mu \nu} H
$$

The QCD corrections to the squark loop are then fully determined by the anomalous mass dimension of the squarks $\gamma_{\tilde{Q}}=4 \alpha_{s} /(3 \pi)$ and by the squark contribution to the $\beta$ function $\beta_{\tilde{Q}} / \alpha=2 \alpha / \pi\left(1+4 \alpha_{s} / \pi\right)$ [34. This results into a final rescaling of the lowest-order Lagrangian by factor

$$
\mathcal{M}_{\tilde{Q}}^{\gamma \gamma H} \rightarrow \mathcal{M}_{\tilde{Q}}^{\gamma \gamma H}\left[1+\frac{8}{3} \frac{\alpha_{s}}{\pi}\right]
$$

This has to be compared with the case of heavy quark loops where the QCD correction gives a rescaling factor [12]

$$
\mathcal{M}_{Q}^{\gamma \gamma H} \rightarrow \mathcal{M}_{Q}^{\gamma \gamma H}\left[1-\frac{\alpha_{s}}{\pi}\right]
$$




\section{References}

[1] For a review on the Higgs sector of the SM and the MSSM , see J.F. Gunion, H.E. Haber, G.L. Kane and S. Dawson, The Higgs Hunter's Guide, Addison-Wesley, Reading 1990 .

[2] Y. Okada, M. Yamaguchi and T. Yanagida, Prog. Theor. Phys. 85 (1991) 1;

H. Haber and R. Hempfling, Phys. Rev. Lett. 66 (1991) 1815;

J. Ellis, G. Ridolfi and F. Zwirner, Phys. Lett. 257B (1991) 83;

R. Barbieri, F. Caravaglios and M. Frigeni, Phys. Lett. 258B (1991) 167.

[3] R. Hempfling and A. Hoang, Phys. Lett. B331 (1994) 99;

J.A. Casas, J.R. Espinosa, M. Quiros and A. Riotto, Nucl. Phys. B436 (1995) 3;

M. Carena, J.R. Espinosa, M. Quiros and C. Wagner, Phys. Lett. B355 (1995) 209;

H. Haber, R. Hempfling and A. Hoang, Report CERN-TH/95-216, hep-ph/9609331.

[4] H.E. Haber, CERN-TH/95-109 and SCIPP-95/15, Proceedings, Conference on Physics Beyond the Standard Model IV, Lake Tahoe CA 1994; and Perspectives for Electroweak Interactions in $e^{+} e^{-}$Collisions, Ringberg Castle, Tegernsee 1995.

[5] M. Sher, Phys. Lett. B317 (1993) 159; addendum, ibid. B331 (1994) 448;

G. Altarelli and G. Isidori, Phys. Lett. B337 (1994) 141.

[6] J.A. Casas, J.R. Espinosa and M. Quiros, Phys. Lett. B342 (1995) 171;

M.A. Díaz, T.A. ter Veldhuis and T.J. Weiler, Report VAND-TH-94-14-UPD, hepph/9512229.

[7] G. Kane, C. Kolda and J. D. Wells, Phys. Rev. Lett. 70 (1993) 2685;

D. Comelli and J.R. Espinosa, Report DESY 96-133, hep-ph/9607400.

[8] H. Georgi et al., Phys. Rev. Lett. 40 (1978) 692.

[9] F. Wilczek, Phys. Rev. Lett. 39 (1977) 1304;

R.N. Cahn, M.S. Chanowitz and N. Fleishon, Phys. Lett. B82 (1979) 113;

L. Bergstrom and G. Hulth, Nucl. Phys. B259 (1985) 137; err. B276 (1986) 744.

[10] J. Ellis, M.K. Gaillard and D.V. Nanopoulos, Nucl. Phys. B106 (1976) 292;

A. I. Vainshtein, M. Voloshin, V. Zakharov and M. Shifman, Sov. J. Nucl. Phys. 30 (1979) 711.

[11] A. Djouadi, M. Spira and P.M. Zerwas, Phys. Lett. B264 (1991) 440;

S. Dawson, Nucl. Phys. B359 (1991) 283;

D. Graudenz, M. Spira and P.M. Zerwas, Phys. Rev. Lett. 70 (1993) 1372. 
[12] M. Spira, D. Graudenz, A. Djouadi and P.M. Zerwas, Nucl. Phys. B453 (1995) 17.

[13] M. Hildreth, T.L. Barklow and D.L. Burke, Phys. Rev. D49 (1994) 3441.

[14] A. Djouadi, V. Driesen, W. Hollik and A. Kraft, KA-TP-29-1996.

[15] For a recent review on Higgs physics at future hadron and $e^{+} e^{-}$colliders see e.g., A. Djouadi, Int. J. Mod. Phys. A10 (1995) 1.

[16] A. Barroso, J. Pulido and J. C. Romao, Nucl. Phys. B267 (1985) 509;

A. Abbassadi, D. Bowser-Chao, D. Dicus and W. Repko, Phys. Rev. D52 (1995) 3919;

A. Djouadi, V. Driesen, W. Hollik and J. Rosiek, KA-TP-21-96, hep-ph/9609420.

[17] B. Mele, talk given at Physics and Detectors for a Linear Collider, DESY Hamburg, November 1996.

[18] J.F. Gunion and H.E. Haber, Phys. Rev. D48 (1993) 5109.

[19] D.L. Borden, D.A. Bauer and D.O. Caldwell, Phys. Rev. D48 (1993) 4018.

[20] I. Watanabe, Report OCHA-PP-58, hep-ph/9504226.

[21] D.L. Borden, V. A. Khoze, W.J. Stirling and J. Ohnemus, Phys. Rev. D50 (1994) 4499.

[22] M. Baillargeon, G. Bélanger and F. Boudjema, Phys. Rev. D51 (1995) 4712.

[23] I.F. Ginzburg, G. Kotkin, V. Serbo and V.I. Telnov, Nucl. Instrum. Meth. 205 (1983) 47 and 219 (1984) 5;

V.I. Telnov, Nucl. Instrum. Meth. A294 (1990) 72 and A335 (1995) 3.

[24] I.F. Ginzburg and V. Serbo, in Proceedings of the 1993 Workshop in Physics and Experiments with Linear $e^{+} e^{-}$Colliders, Waikaloa, Hawaii. Eds. F.A. Harris et al., World Scientific 1993.

[25] H. Murayama, I. Watanabe and K. Hagiwara, HELAS: HELicity Amplitude Subroutines for Feynman Diagram Evaluations, KEK Report 91-11, January 1992.

[26] For recent discussions in the MSSM see e.g.,

G. L. Kane, G. D. Kribs, S. P. Martin and J. D. Wells, Phys. Rev. D53 (1996) 213;

B. Kileng, P. Osland and P.N. Pandita, Report NORDITA-95-48-P (1995).

[27] For a recent update of the Higgs decay widths and branching ratios, see e.g.: A. Djouadi, M. Spira and P. Zerwas, Z. Phys. C70 (1996) 427. 
[28] V. Barger, M. Berger, A. Stange and R. Phillips, Phys. Rev. D45 (1992) 4128;

A. Brignole and F. Zwirner, Phys. Lett. B299 (1993) 72.

[29] J. Ellis and S. Rudaz, Phys. Lett. B128 (1983) 248;

A. Bartl et al., Report UWTHPH-1996-18, hep-ph/9603410.

[30] F. Abe et al. (CDF Coll.), Phys. Rev. Lett. 76 (1996) 2006;

S. Abachi et al. (D0 Coll.), Phys. Rev. Lett. 76 (1996) 2222.

[31] H.E. Haber and G. Kane, Phys. Rep. 117 (1985) 75;

J.F. Gunion and H.E. Haber, Nucl. Phys. B272 (1986) 1.

[32] J.M. Frère, D.R.T Jones and S. Raby, Nucl. Phys. B222 (1983)11;

M. Claudson, L. Hall and I. Hichliffe, Nucl. Phys. B228 (1983) 501;

J.F. Gunion and H.E. Haber and M. Sher, Nucl. Phys. B306 (1998) 1;

M. Drees and K. Hagiwara, Phys. Rev. D42 (1990) 1709.

[33] S. Dawson, A. Djouadi and M. Spira, Phys. Rev. Lett. 77 (1996) 17;

B. Kniehl and M. Spira, Z. Phys. C69 (1995) 77.

[34] W. Caswell, Phys. Rev. Lett. 33 (1974) 244;

D.R.T. Jones, Phys. Rev. D25 (1982) 581;

M. Eihorn and D.R.T. Jones, Nucl. Phys. B196 (1982) 475. 


\section{Figure Captions}

Fig. 1: Feynman diagrams contributing to the Higgs decay into two photons in the MSSM.

Fig. 2: The masses of the three MSSM Higgs bosons $h, H$ and $H^{ \pm}$as a function of the pseudoscalar mass $M_{A}$ for $\tan \beta=1.1,1.6,5$ and 50 ; the common squark mass is set to $m_{\tilde{q}}=250 \mathrm{GeV}$ (a) and $1 \mathrm{TeV}$ (b) and the squark mixing is neglected.

Fig. 3: The amplitudes for the contribution of the $W$ boson loop (a) and of the $t, b$ loops (b) as a function of $M_{h}$ in the SM [dashed lines] and MSSM [solid lines]; $M_{A}$ is fixed to $250 \mathrm{GeV}$. We have used $m_{\tilde{q}}=250 \mathrm{GeV}$ and neglected squark mixing.

Fig. 4: The amplitudes for the contribution of the charged Higgs boson loop (a) and of the slepton and squark (except stop) loops (b) as functions of the loop masses for $\tan \beta=1.6,5$ and 50. We have neglected squark mixing and for $H^{ \pm}$and slepton loops we used $m_{\tilde{q}}=250 \mathrm{GeV}$; for sfermion loops we have set $M_{A}=250 \mathrm{GeV}$.

Fig. 5: Contours in the $\left(m_{t}^{L R}, m_{\tilde{t}_{1}}\right)$ plane, for which: (a) the contribution of the stop loops to the $h \gamma \gamma$ coupling is $\left|A_{\tilde{t}}\right|=2,1,0.5$ and 0.2 , and (b) the heavier top squark mass $m_{\tilde{t}_{2}}$ and the common mass of the scalar partners of light quarks $m_{\tilde{q}}$.

Fig. 6: The separate contributions of the lightest (a) and the heaviest (b) top squark loops to the form factor $A_{\tilde{t}}$ as a function of $m_{t}^{L R}$ and $m_{\tilde{t}_{1}}$.

Fig. 7: Contours in the $\left(M_{2}, \mu\right)$ plane for $\tan \beta=1.6$ (a) and $\tan \beta=50$ (b) for which the contribution of the chargino loops to the $h \gamma \gamma$ coupling is $\left|A_{\chi}\right|=0.1,0.2,0.3$ and 0.5. Also included are the contours for which the lightest chargino mass is $m_{\chi_{1}^{+}}=90$ and $250 \mathrm{GeV}$.

Fig. 8: The deviations of the SUSY Higgs coupling to two photons from the Standard Model value [in \%] for two values of $\tan \beta=1.6$ and 50 and the loops masses $m_{i}=250$ and $400 \mathrm{GeV}$. (a) Deviations due to the chargino loops as a function of $M_{2}$ for both signs of $\mu$, and (b) deviations due to the top squark loops as a function of $m_{t}^{L R}$. 

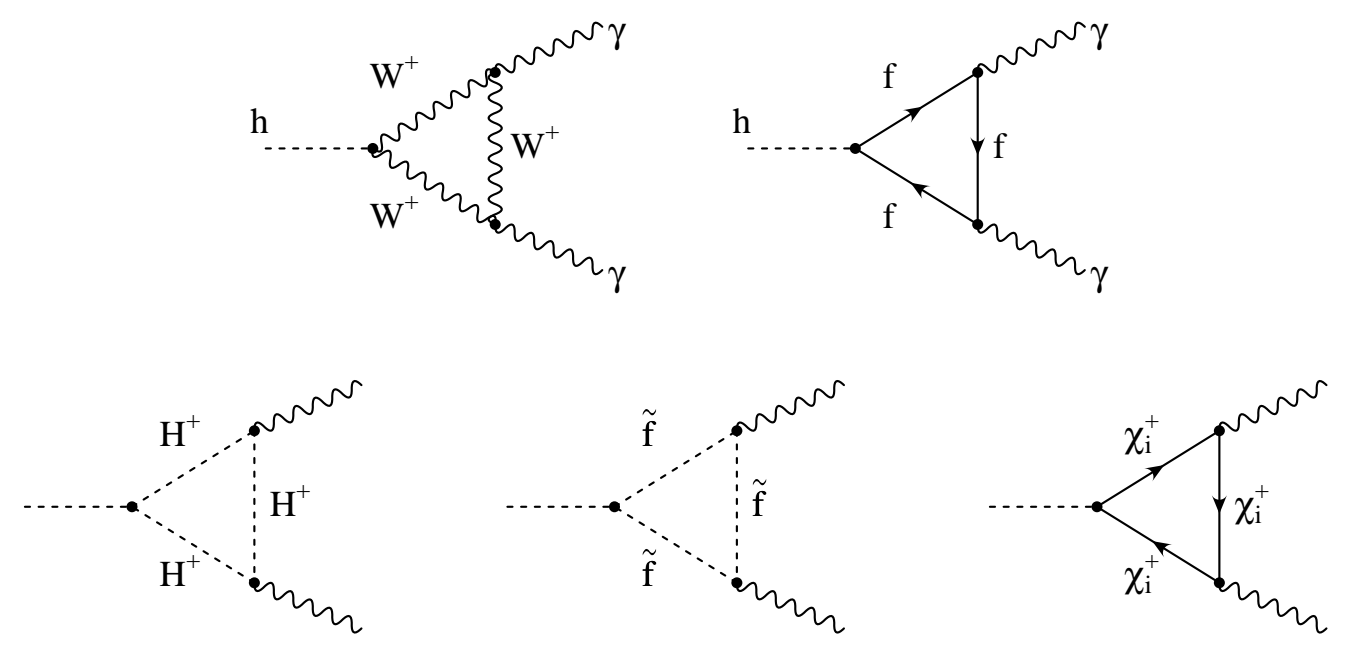

Fig. 1 


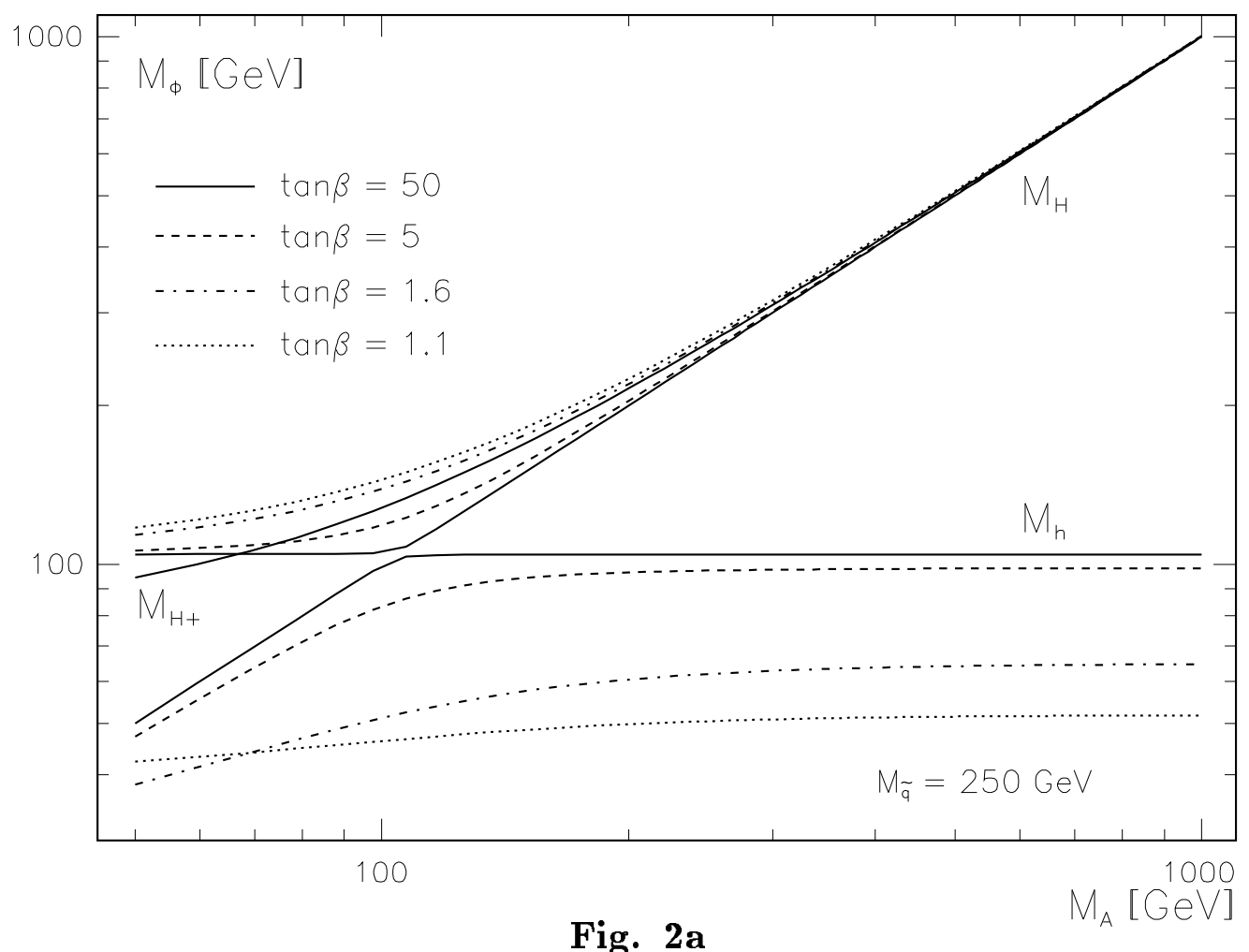

Fig. 2a

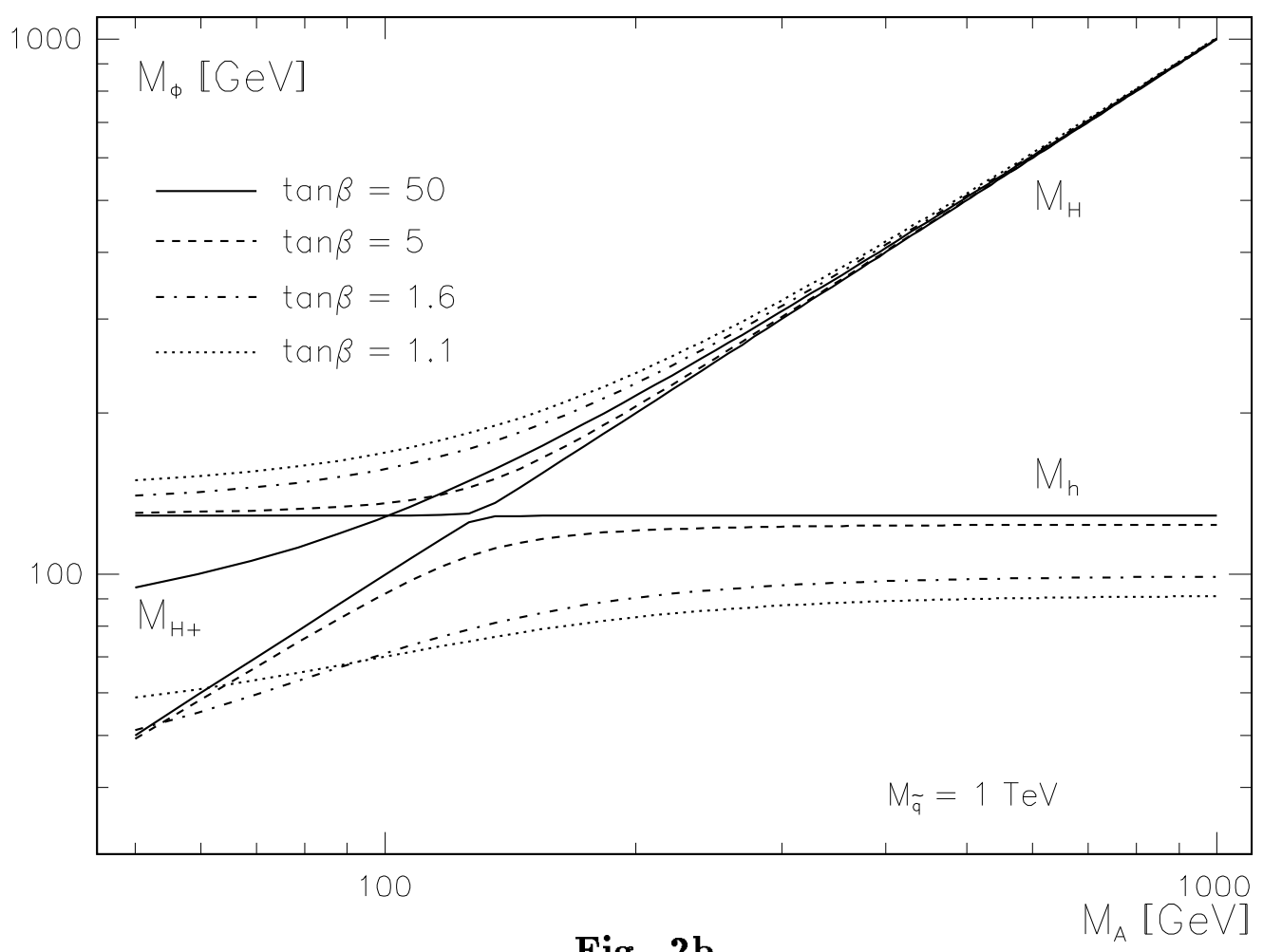

Fig. 2b 


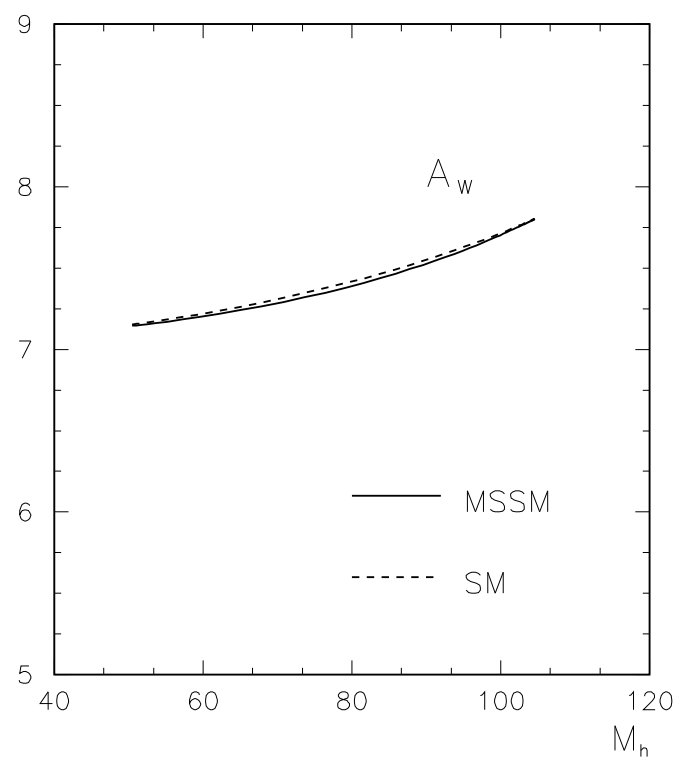

Fig. 3a
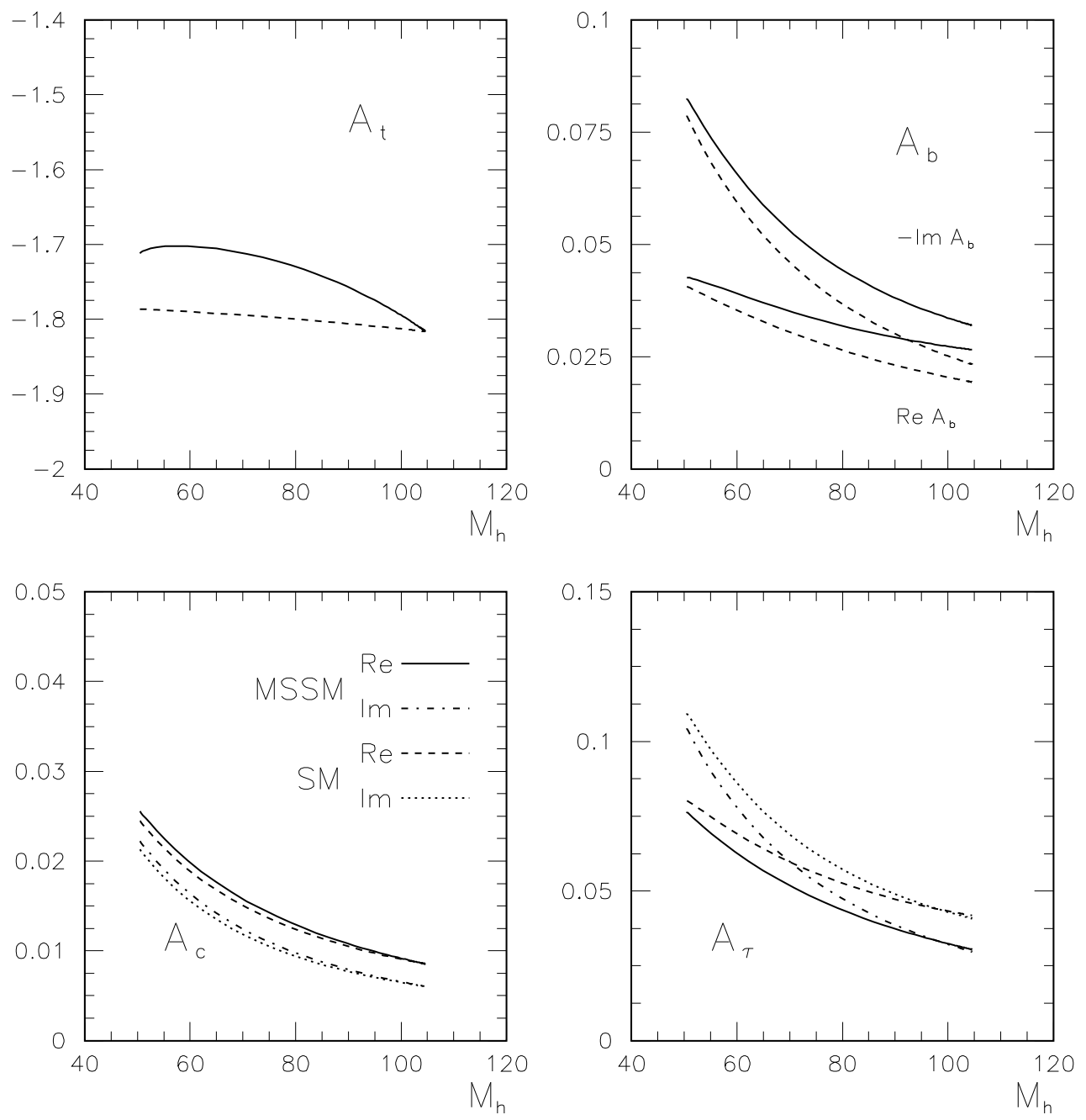

Fig. 3b 


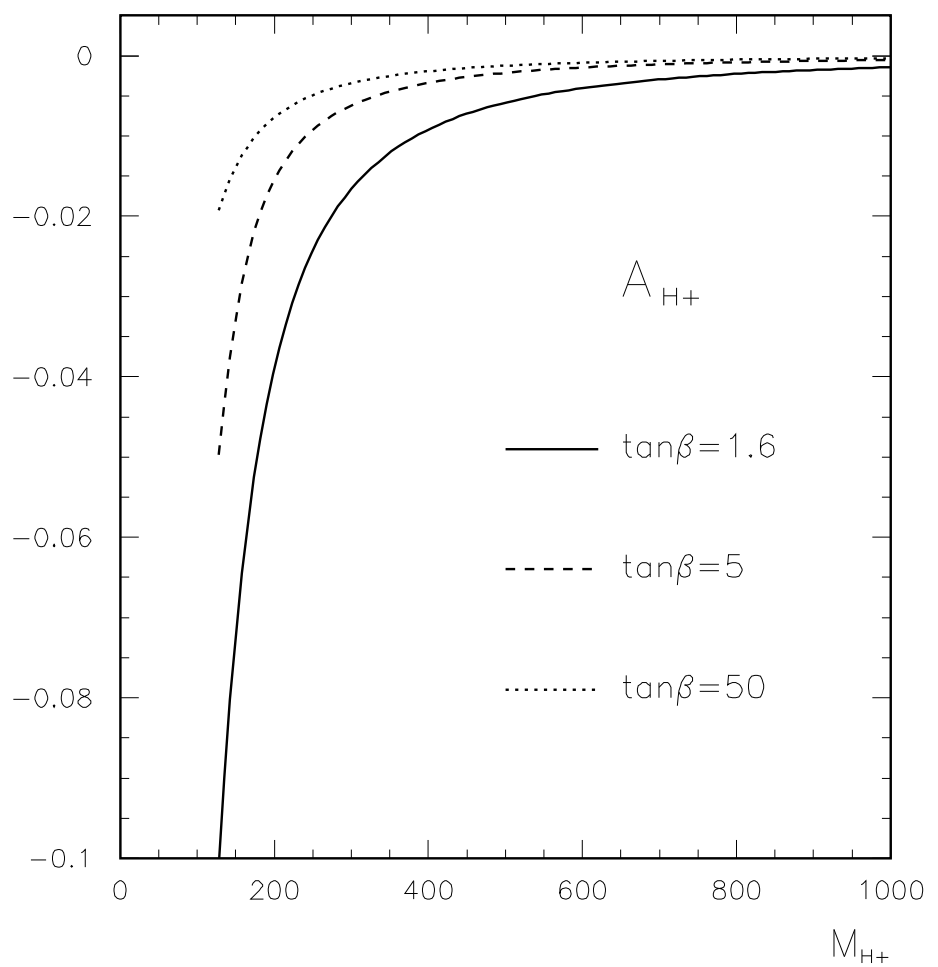

Fig. 4a
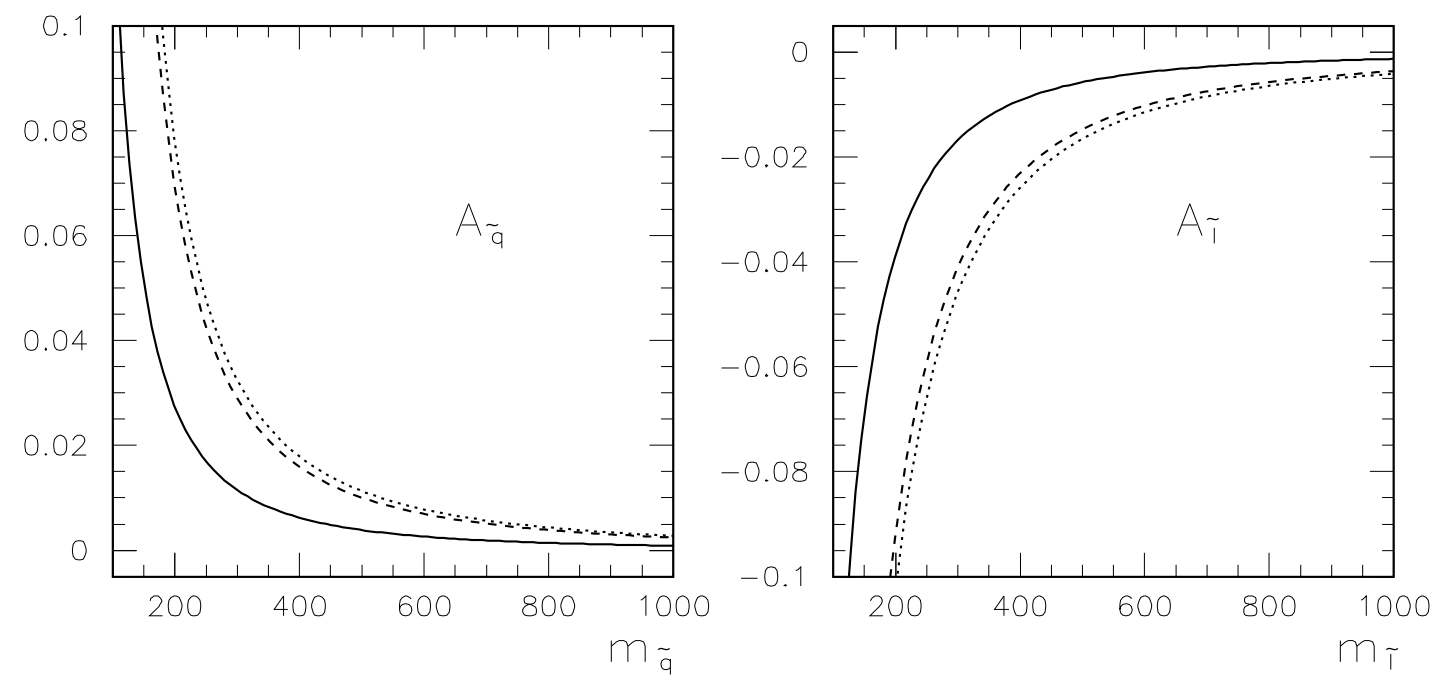

Fig. $4 b$ 


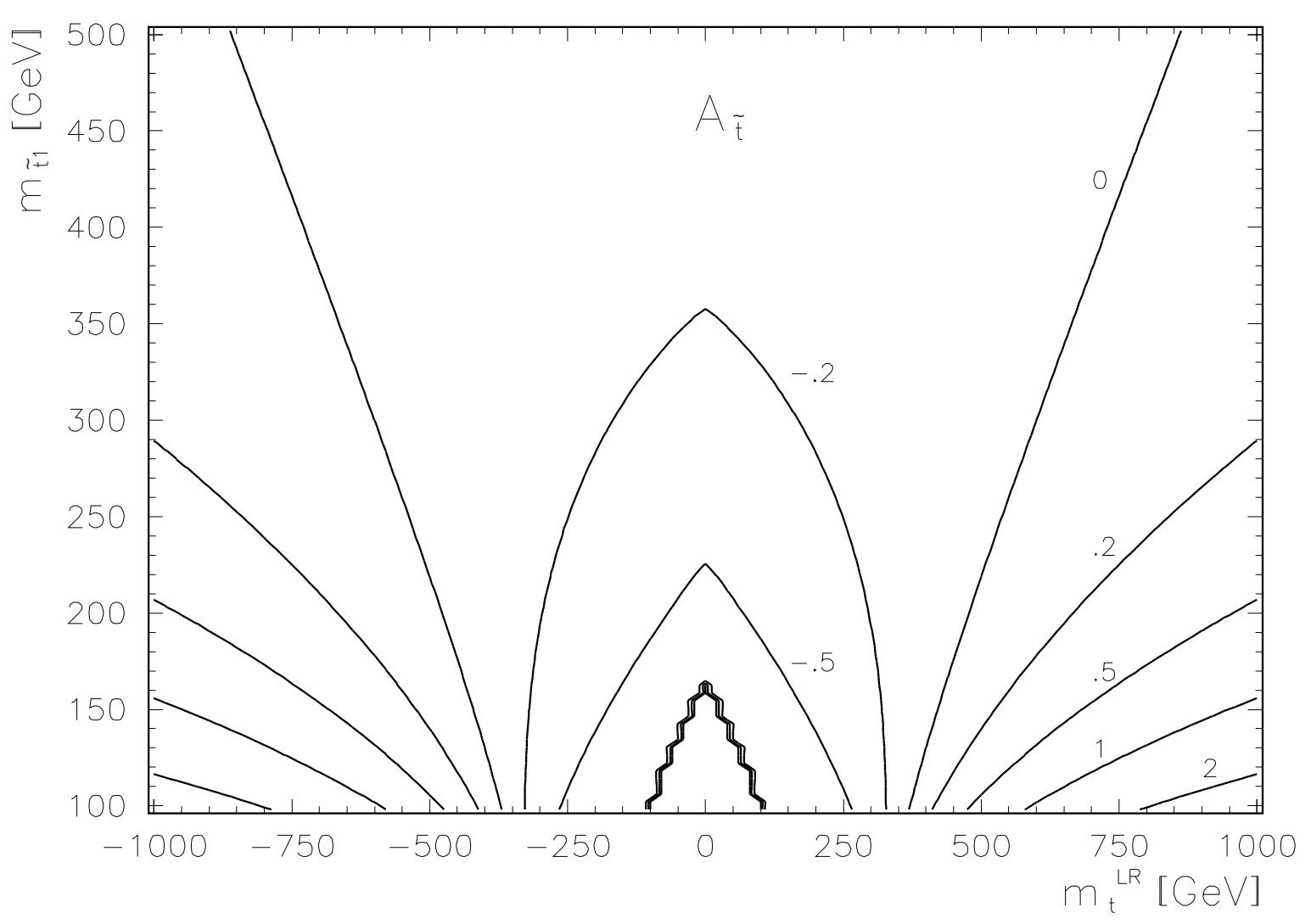

Fig. 5a
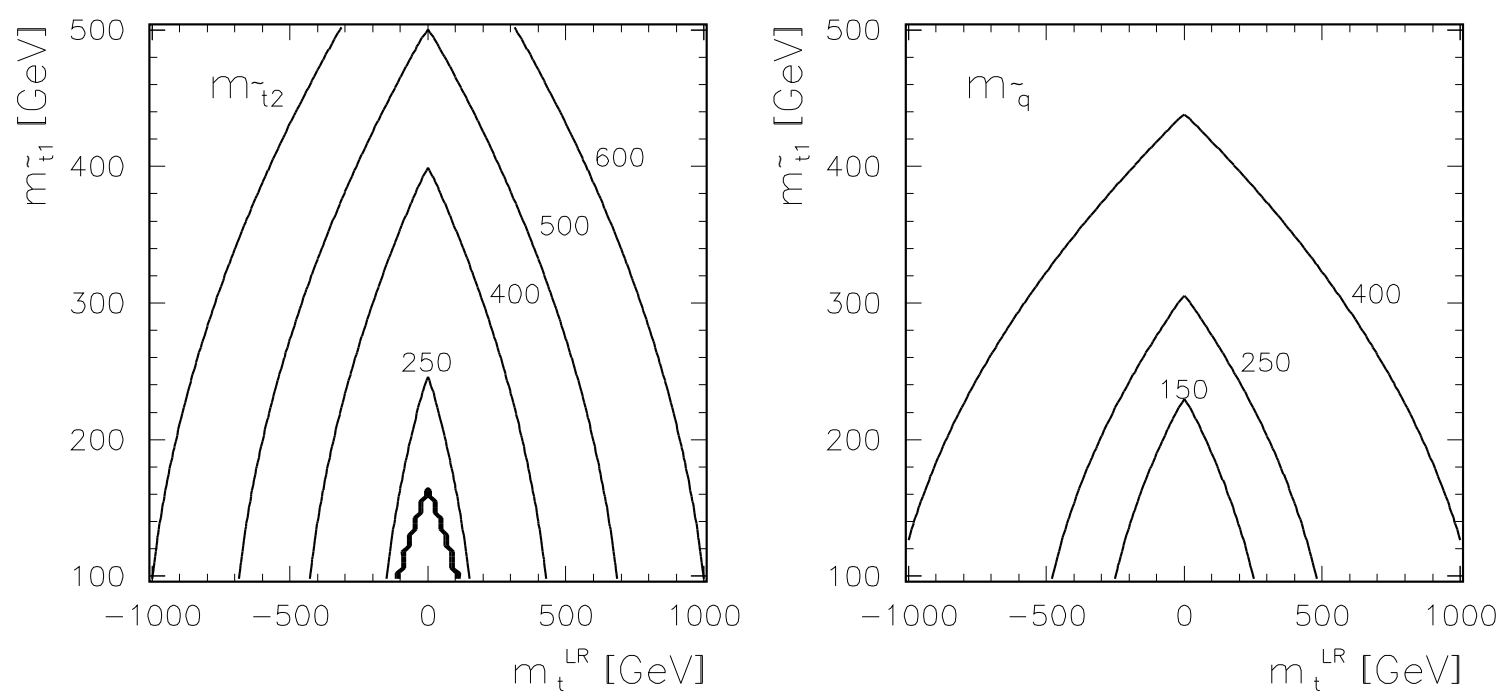

Fig. 5b 

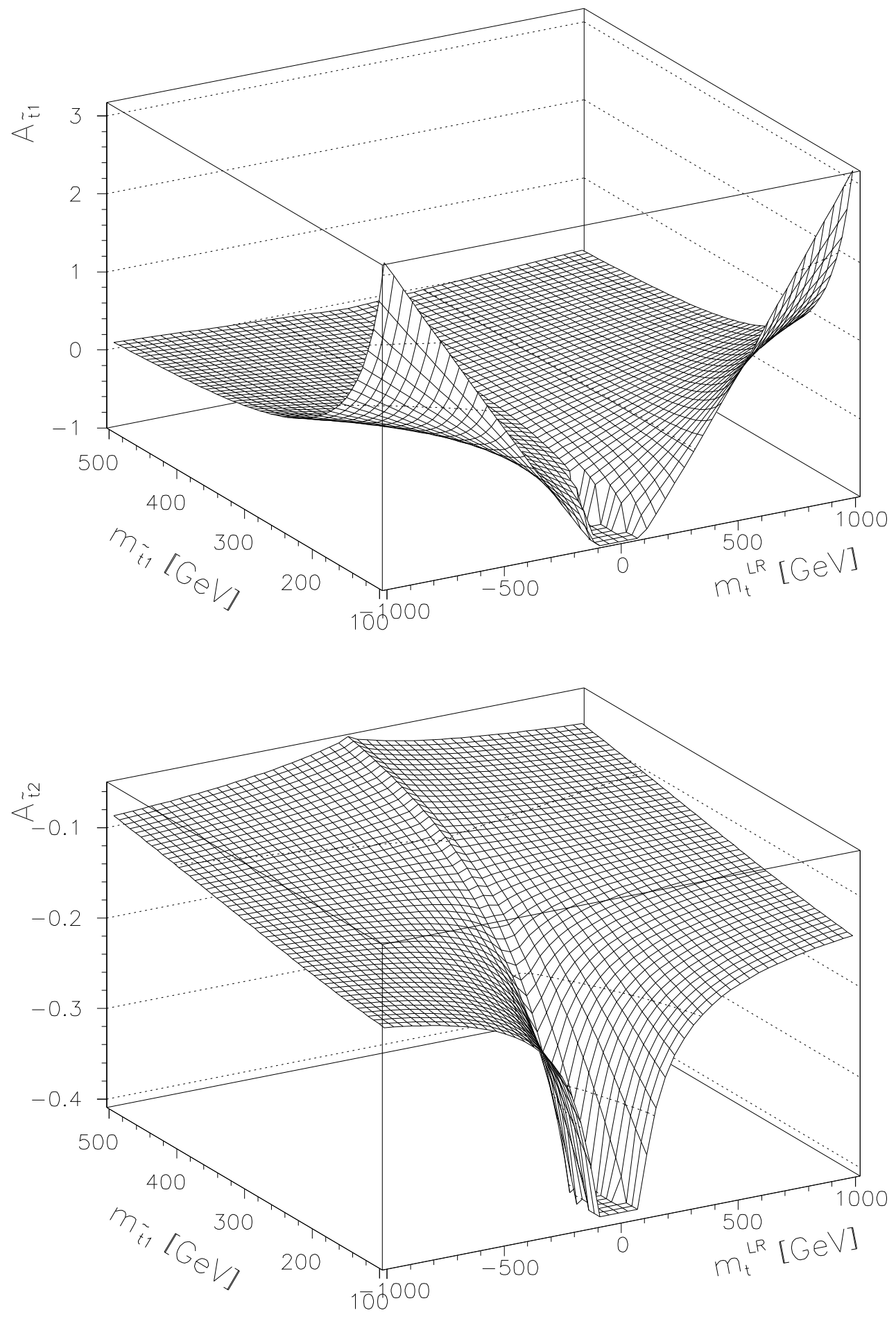

Fig. 6 


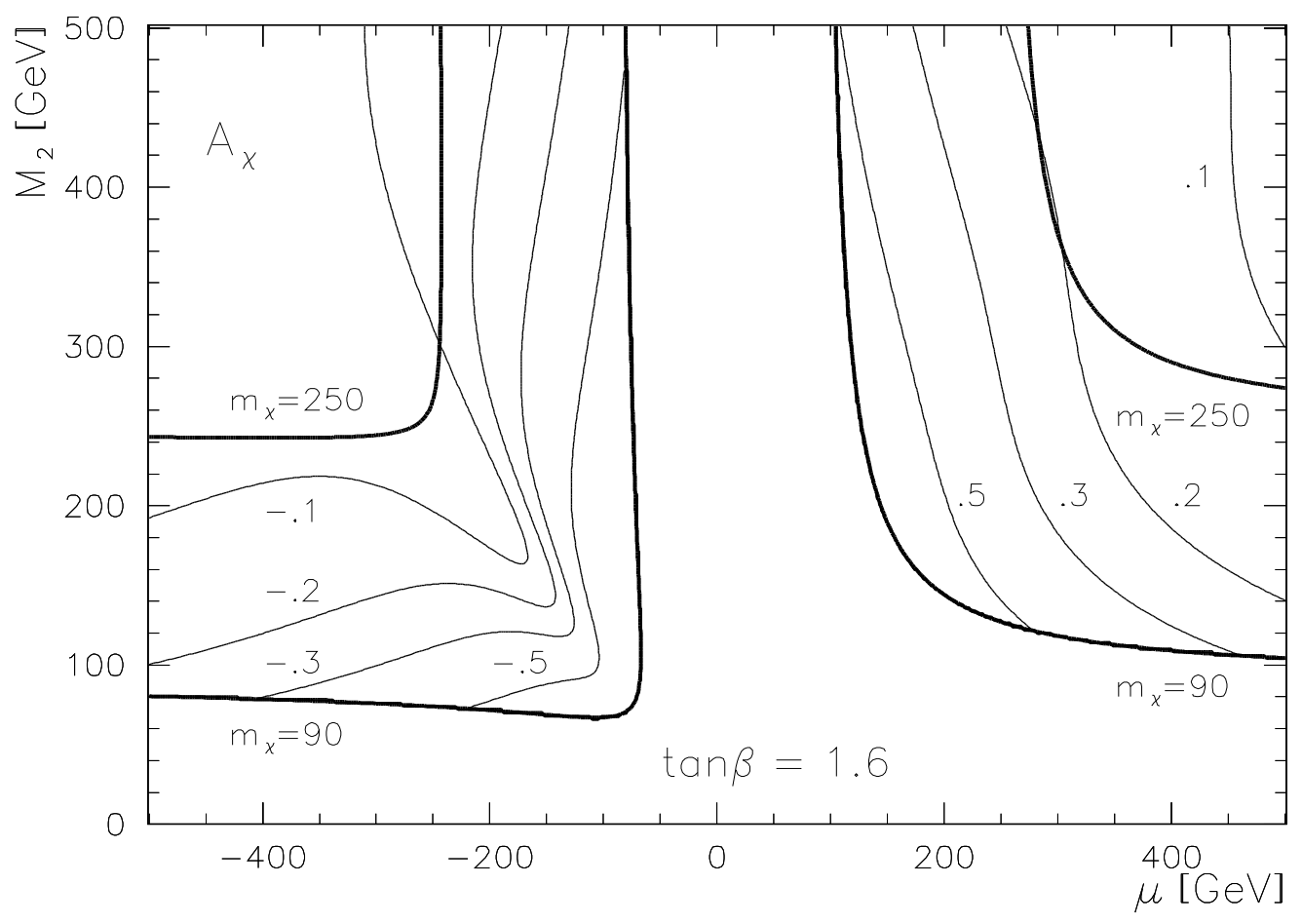

Fig. 7a

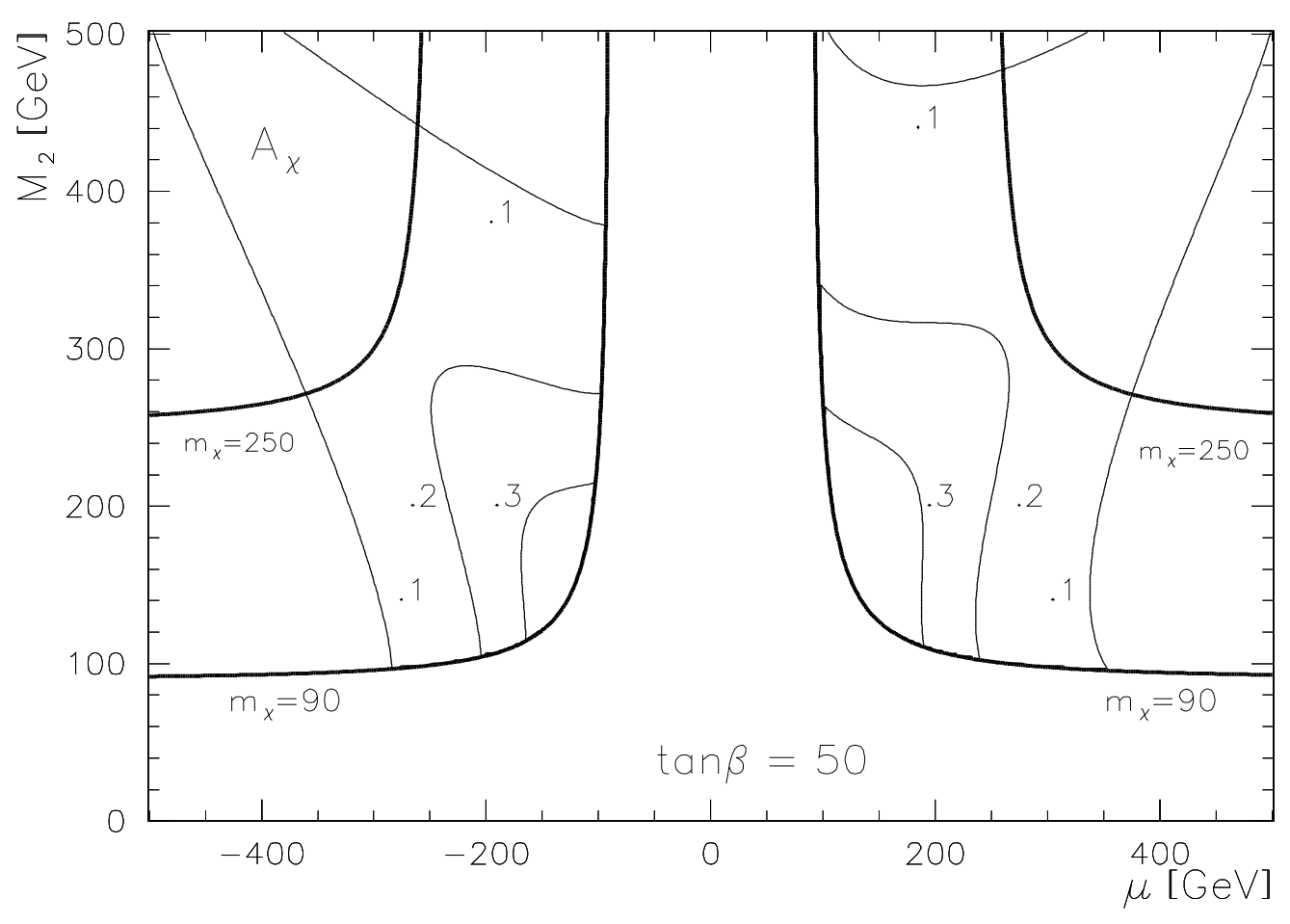

Fig. 7b 

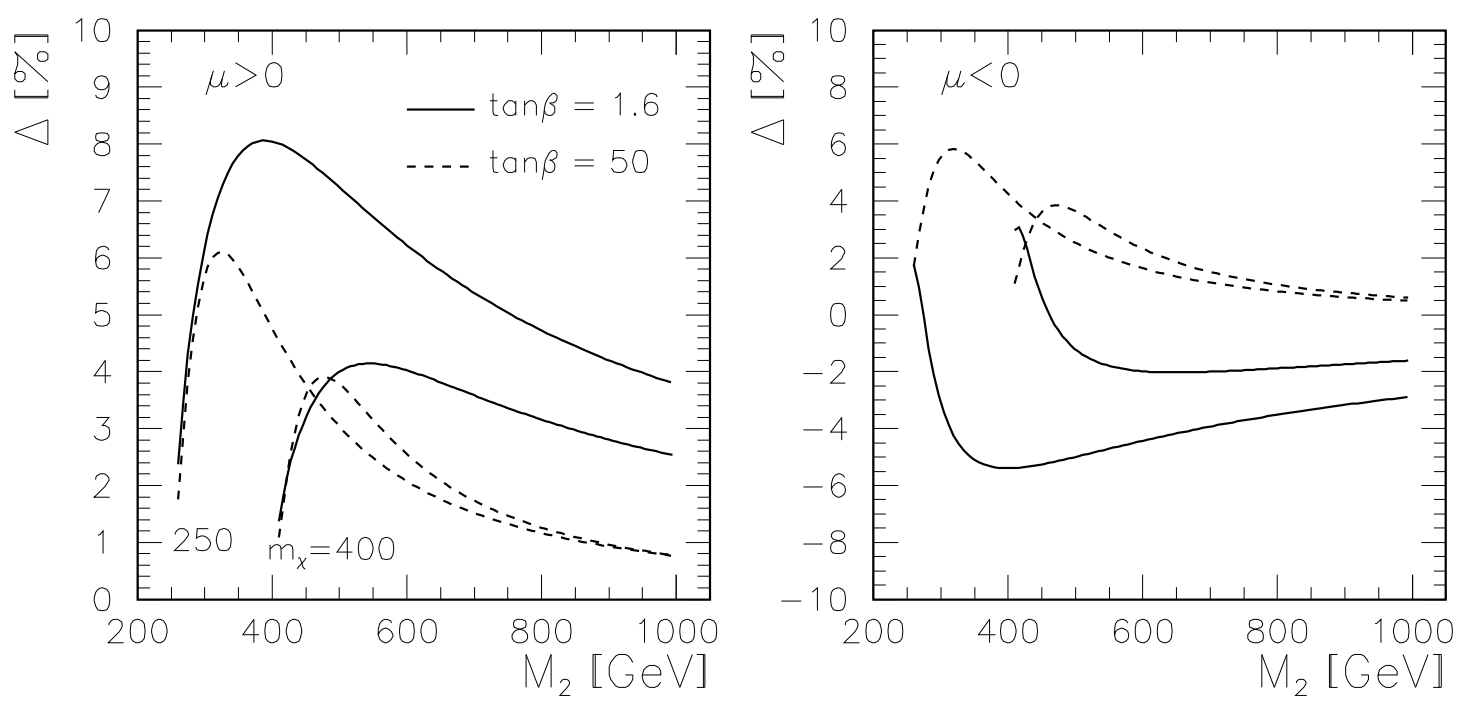

Fig. $8 \mathbf{a}$

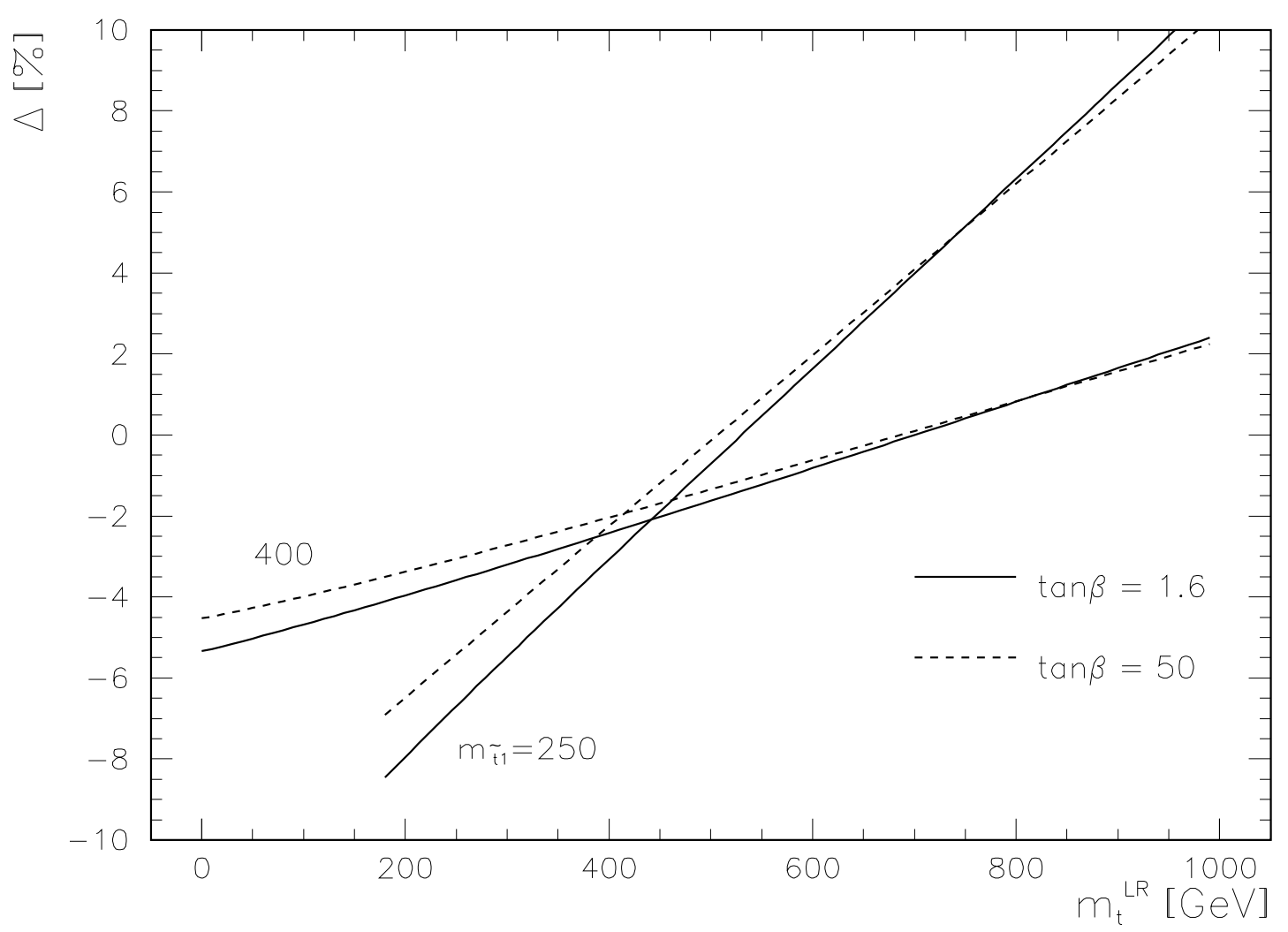

Fig. $8 b$ 